\title{
Star formation history of Canis Major R1
}

\section{Wide-Field X-ray study of the young stellar population ${ }^{\star}$}

\author{
J. Gregorio-Hetem ${ }^{1}$, T. Montmerle ${ }^{2}$, C. V. Rodrigues ${ }^{3}$, E. Marciotto ${ }^{1}$, T. Preibisch ${ }^{4}$, and H. Zinnecker ${ }^{5}$ \\ 1 Universidade de São Paulo, IAG, Departamento de Astronomia, Brazil \\ e-mail: jane@astro.iag.usp.br \\ ${ }^{2}$ Laboratoire d'Astrophysique de Grenoble, Université Joseph Fourier-CNRS, France \\ 3 Instituto Nacional de Pesquisas Espaciais, Divisão de Astrofísica, São José dos Campos, SP, Brazil \\ 4 Universitäts-Sternwarte München, Scheinerstr. 1, 81679 München, Germany \\ 5 Astrophysikaliches Institut Potsdam, Germany
}

Received 24 March 2009 / Accepted 28 July 2009

ABSTRACT

\begin{abstract}
Aims. The CMa R1 star-forming region contains several compact clusters as well as many young early-B stars. It is associated with a well-known bright rimmed nebula, the nature of which is unclear (fossil HII region or supernova remnant). To help elucidate the nature of the nebula, our goal was to reconstruct the star-formation history of the CMa R1 region, including the previously unknown older, fainter low-mass stellar population, using X-rays.

Methods. We analyzed images obtained with the ROSAT satellite, covering $\sim 5$ sq. deg. Complementary VRI photometry was performed with the Gemini South telescope. Colour-magnitude and colour-colour diagrams were used in conjunction with pre-main sequence evolutionary tracks to derive the masses and ages of the X-ray sources.

Results. The ROSAT images show two distinct clusters. One is associated with the known optical clusters near $\mathrm{Z}$ CMa, to which $\sim 40$ members are added. The other, which we name the "GU CMa" cluster, is new, and contains $\sim 60$ members. The ROSAT sources are young stars with masses down to $M_{\star} \sim 0.5 M_{\odot}$, and ages up to $10 \mathrm{Myr}$. The mass functions of the two clusters are similar, but the GU CMa cluster is older than the cluster around Z CMa by at least a few Myr. Also, the GU CMa cluster is away from any molecular cloud, implying that star formation must have ceased; on the contrary (as already known), star formation is very active in the Z CMa region.
\end{abstract}

Key words. stars: pre-main sequence - X-rays: stars - infrared: stars - ISM: clouds

\section{Introduction}

$\mathrm{CMa} \mathrm{R} 1$ is an association of bright stars and clusters distributed around and in the vicinity of the long $\left(200^{\prime}\right)$ arc-shaped ionized reflection nebula Sh2-296 (Sharpless 1959: $l=224.6^{\circ}$, $b=-2^{\circ}$ ), located at a distance $d \sim 1 \mathrm{kpc}$ (Shevchenko et al. 1999; Kaltcheva \& Hilditch 2000). In the absence of conspicuous exciting early-type stars inside the arc, the source of ionization is still being debated. The CMa R1 nebulae are found within the boundaries of the OB1 association, which approximately are: $222^{\circ}<l<226^{\circ}$ and $-3.4^{\circ}<b<+0.7^{\circ}$ (Ruprecht 1966). Clariá (1974a,b) studied the space distribution of $O$ and $B$ stars, based on $U B V$ photometry obtained for 247 stars. The estimated $E(B-V)$ colour excess confirms the existence of a group of young OB stars together with excited gas and obscuring matter, which belong to the $\mathrm{CMa} \mathrm{OB} 1$ association. Clariá used these data to derive an age of $3 \mathrm{Myr}$.

* Based in part on observations obtained at the Gemini Observatory, which is operated by the Association of Universities for Research in Astronomy, Inc., under a cooperative agreement with the NSF on behalf of the Gemini partnership: the National Science Foundation (United States), the Science and Technology Facilities Council (United Kingdom), the National Research Council (Canada), CONICYT (Chile), the Australian Research Council (Australia), Ministério da Ciência e Tecnologia (Brazil) and Ministerio de Ciencia, Tecnología e Innovación Productiva (Argentina).
In the early years of the "propagating star formation" models (Elmegreen \& Lada 1977), Herbst \& Assousa (1977) suggested that the "CMa R1 ring" (Sh2-296) could be an old supernova remnant (SNR), which was inducing star formation in CMa $\mathrm{R} 1$. Indeed, linear polarization observations are consistent with a model of compression by a supernova shock (Vrba, Baierlein \& Herbst 1987). Alternatively, Reynolds \& Ogden (1978) proposed that the nebula and star formation were induced by strong stellar winds, or by an evolving, "fossil" HII region, as also suggested by Blitz (1980) and Pyatunina \& Taraskin (1986). However, because they are found to be in pressure equilibrium with the parent molecular cloud once they have opened a cavity (see the example of M 17, Townsley et al. 2003; or Orion, Güdel et al. 2008), stellar winds cannot compress the surrounding medium and induce star formation. On the other hand, we now know many examples of HII "bubbles" apparently triggering star formation near their edges (e.g., Deharveng et al. 2008; Zavagno et al. 2007), but these bubbles have well-defined exciting stars.

To this day, the nature of the Sh2-296 nebula remains a mystery, yet we see that star formation is going on in the vicinity of the nebula. The location of the Sh2-296 arc and of other visible sites of star formation, in particular the associated nebulae Sh2-292 (IC 2177) and Sh2-297 (HD 53623), with respect to the cold dust distribution, are shown in Fig. 1, in the form of IRAS $100 \mu \mathrm{m}$ contours, superimposed on a DSS optical (R) image. It is clear that the sites of star formation correspond to the largest 


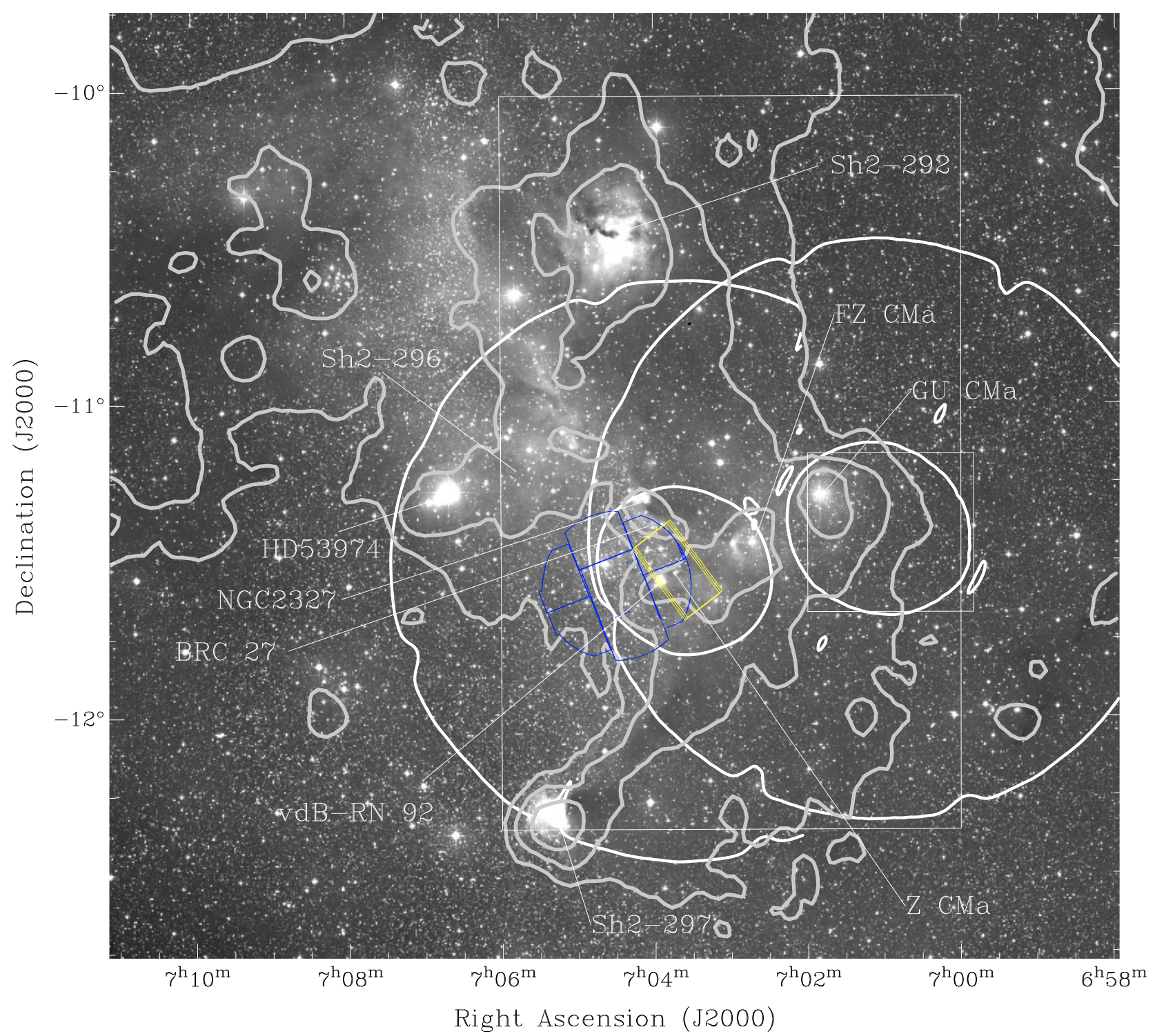

Fig. 1. Finding chart of the CMa OB1/R1 region. Far-infrared IRAS-ISIS contours superimposed on a $3 \times 3$ square degrees optical image extracted from the Digitized Sky Survey. The grey contours correspond to data obtained at $100 \mu \mathrm{m}$, tracing the dust component. The field-of-view of X-ray observations are indicated for: ROSAT (white lines); XMM-Newton (blue) and Chandra (yellow). The large rectangular white box shows the area covered by the optical survey of OB stars by Shevchenko et al. (1999), while the small square box including GU CMa corresponds to the area where Gemini observations of unresolved X-ray sources were carried out. Objects discussed in the text are labelled by their names. North is up and east is to the left.

dust column densities, mainly distributed along the edge of the arc.

The molecular gas around CMa R1 has also been studied in the course of millimeter surveys of the third galactic quadrant. The ${ }^{12} \mathrm{CO}(1 \rightarrow 0)$ Columbia survey of May et al. (1988), with a spatial resolution of $0.5^{\circ}$, reveals the extent, down to low densities, of the molecular clouds in the $\mathrm{CMa} \mathrm{R} 1$ region. Taking into account the relatively low spatial resolution of the survey, the ${ }^{12} \mathrm{CO}$ contours match the IRAS $100 \mu \mathrm{m}$ maps. The densest regions are outlined in the ${ }^{13} \mathrm{CO}(1 \rightarrow 0)$ Nanten survey of Kim et al. (2004), which has a better spatial resolution (8.8' spacing). These regions are the "backbone" of molecular clouds, where the earliest stages of star formation can be found. Here they are not so well correlated with IRAS maps, but, as expected, underlie the other nebulae. These CO maps, with which we will compare the young stars distribution, give precious clues about the star formation history of the region. (See below, Sect. 6 and Fig. 12, for further discussion.)
Earlier works already contain evolutionary information about the stellar population in the CMa R1 region. A survey of early-type stars was done by Shevchenko et al. (1999) covering a 4 sq. deg. rectangular area that overlaps the Sh2-296 arc, revealing 88 members of the CMa R1 association (74 B stars, no $\mathrm{O}$ star) with ages between 8 and $0.5 \mathrm{Myr}$, apparently suggesting an extended period of star formation activity that is still ongoing.

Shevchenko et al. (1999) also point out that two other bright B stars, GU CMa and FZ CMa, seem to be older than the association and may not have been formed in the same star formation episode. Other early B stars associated with Sh2-296 include Z CMa, a double system (Koresko et al. 1991; Haas et al. 1992) containing a FU Orionis object (Hartmann et al. 1989) and a Herbig Be star, with a common age of about 0.3 Myr (van den Ancker et al. 2004).

In addition, three embedded stellar clusters, NGC 2327, BRC 27, and VdB-RN92, are found along the outer rim of Sh2296 , in the vicinity of Z CMa. These clusters were studied by 
Soares \& Bica (2002, 2003), who used JHK data from 2MASS ("The Two Micron All Sky Survey", Skrutskie et al. 2006). Based on colour-magnitude diagrams, they estimated ages of 1.5 Myr (NGC2327 and BRC27) and 5-7 Myr (VdB-RN92), spanning the same age range as the sample of Shevchenko et al. (1999).

We note, however, that such surveys, by construction, miss the most numerous, fainter low-mass $\left(<2 M_{\odot}\right)$ star population, which may have a different spatial distribution, as well as different ages. The most efficient method to find young stars including low-mass ones is to use X-rays, the lower-mass limit being only a question of sensitivity for a given instrument, i.e., of exposure time and distance to the star-forming region. The reasons are twofold: (i) young stars of all masses produce X-rays (except perhaps A stars, see, e.g., Stelzer et al. 2005), independently of their circumstellar environment: massive stars via shocks in their winds, with a typical X-ray-to-bolometric luminosity ratio $L_{\mathrm{X}} / L_{\mathrm{bol}} \sim 10^{-7}$, low-mass stars via their magnetic activity, with $L_{\mathrm{X}} / L_{\text {bol }} \sim 10^{-5}-10^{-3}$; (ii) X-ray satellites have a wide fieldof-view, covering up to several square degrees in one exposure. (For reviews, see Feigelson \& Montmerle 1999; Favata \& Micela 2003; Güdel 2004.)

By having access in this way to a much more complete census of the stellar population, down to low-mass stars, we will show in this paper how we can significantly improve our knowledge of the evolution of star formation in the area, both in time (from colour-colour diagrams) and in space (wide fieldof-view of X-ray telescopes), and thus investigate whether Sh2296, whatever its nature, may have triggered star formation in the CMa R1 region and its vicinity.

More precisely, in order to obtain a stellar sample covering as large a range of masses and ages as possible, we have analyzed archival X-ray data from the PSPC detector aboard the ROSAT satellite, because of its wide Field of view $\left(1^{\circ}\right.$ radius, or an area of $\sim 3$ sq. deg.).

We will proceed along the following steps: (i) identify the $\mathrm{X}$-ray sources, by comparison with data from optical and IR surveys, as well as from dedicated Gemini VRI observations; (ii) put the counterparts on appropriate colour-magnitude diagrams and deduce their evolutionary status (mass and age) using PMS evolution models; (iii) study the spatial distribution of the X-ray sources and relate it to the mass distribution in its vicinity. In future papers, we will "zoom in" towards more restricted regions in the Z CMa region, using XMM-Newton/EPIC (circle of $15^{\prime}$ radius) and Chandra/ACIS (square FOV, $\left.17^{\prime} \times 17^{\prime}\right)$.

Although we have used the XMM-Newton and Chandra source positions, when available, to validate the ROSAT positions in Field 2, we defer a more detailed analysis of these fields (source luminosities, spectra, etc.) to a future study (see preliminary results by Rojas et al. 2006) for several reasons: (i) as shown in Fig. 1, the XMM-Newton and Chandra observations cover a much smaller area than the ROSAT fields; (ii) the $50 \mathrm{ks} X M M$ Newton total exposure is heavily affected by solar flares, and thus requires a detailed treatment to recover as much information as possible; (iii) in contrast, the Chandra observations are much more sensitive (total exposure of $80 \mathrm{ks}$ in two observations) and thus contain many sources which ROSAT is unable to detect in Field 2: obviously this would distort the comparison between the two ROSAT fields.

This paper is therefore organized as follows. Section 2 describes archival ROSAT/PSPC observations of the CMa R1 region and the general characteristics of the $\mathrm{X}$-ray data. Stellar identifications based on optical and near-IR counterparts searched in published catalogues are described in Sect. 3. The
Gemini observations, which were obtained specifically to search for additional counterparts in the case of unresolved X-ray sources, and their analysis, are presented in Sect. 4. Ages and masses of the ROSAT sources are derived from colour-magnitude diagrams in Sect. 5.

The last section gives a summary of the results, followed by a discussion and conclusions. Appendix A gives details on the ROSAT data analysis, and Appendix B gives complementary results of the Gemini data analysis.

\section{Search for young stars based on X-ray data}

The power of X-ray observations to discover large samples of PMS stars has been illustrated in a variety of star-forming regions. In particular, following up on pioneering ROSAT observations, intermediate to distant clouds have been covered by Chandra: the Orion nebula (Garmire et al. 2000; Feigelson et al. 2002, 2003; Flaccomio et al. 2003), part of the Rosette nebula and molecular cloud, together with M17 (Townsley et al. 2003; Wang et al. 2008, 2009); Mon R2 (Kohno et al. 2002); RCW38 (Wolk et al. 2002); M16 (Linsky et al. 2007), and by XMMNewton: M8 (Rauw et al. 2002); Carina (Albacete Colombo et al. 2003); Vela OB2 (Jeffries et al. 2009); Orion (López-Santiago \& Caballero 2008); and others. Up to thousands of point X-ray sources per cloud are detected, almost all identified with young stars, down to the brown dwarf regime. More than 1600 X-ray sources were detected in the Orion Nebula Cluster during an exceptionally deep survey ( 1 Ms), the "Chandra Orion Ultradeep Project" (COUP: Getman et al. 2005a,b), and several hundred others have been found in the recent "XMM-Newton Extended Survey of Taurus" (XEST: Güdel et al. 2007).

However, in spite of the much improved sensitivity and angular resolution of Chandra and XMM-Newton over ROSAT, there are spatial limitations to these observations. To map a wide area (several square degrees), a mosaic is necessary: for instance, 5 Chandra fields for the Rosette nebula and associated molecular cloud (Wang et al. 2008, 2009), 28 XMM-Newton fields to map the densest molecular clouds of Taurus (Güdel et al. 2007). In the case of Sh2-296, two overlapping archival ROSAT/PSPC fields exist (see Fig. 1), covering $\sim 5$ sq. deg. in total, which we are analyzing in this paper. The present ROSAT work on CMa $\mathrm{R} 1$ is related to a similar study based on observations of two other giant molecular clouds, Monoceros and Rosette (GregorioHetem et al. 1998). The ROSAT images provided the identification and characterization of several dozen sources, making up the bright end of the low- to intermediate-mass young star distribution in the associated clusters. In particular, a useful correlation is found between the intrinsic X-ray luminosity of the sources and their optical and near-IR magnitudes. This correlation was similar to the one found previously by Feigelson et al. (1993) and Casanova et al. (1995) from ROSAT studies of the nearby Chamaeleon I and $\rho$ Ophiuchi clouds, respectively, suggesting a general validity which we will use in the present paper. The main interest in this correlation is that it is weakly dependent on extinction, which is essential for the study of young stars, because of the comparable absorption cross-sections in the near-IR and keV ranges (Ryter 1996).

The two ROSAT observations we analyze are the following. By order of increasing RA, the first Field ("Field 1" hereafter), HEASARC ID RP201011 (PI J.G.H.), pointing axis $\alpha_{\mathrm{J} 2000}=$ $7^{\mathrm{h}} 00^{\mathrm{m}}, \delta_{\mathrm{J} 2000}=-11^{\circ} 30^{\prime}$, has an exposure of $19.7 \mathrm{ks}$. (For preliminary results, see Gregorio-Hetem et al. 2003.) "Field 2", HEASARC ID RP201277 (PI H.Z.), pointing axis $\alpha_{\mathrm{J} 2000}=$ $7^{\mathrm{h}} 04^{\mathrm{m}}, \delta_{\mathrm{J} 2000}=-11^{\circ} 33^{\prime}$, has a much shorter exposure of $4.6 \mathrm{ks}$, 


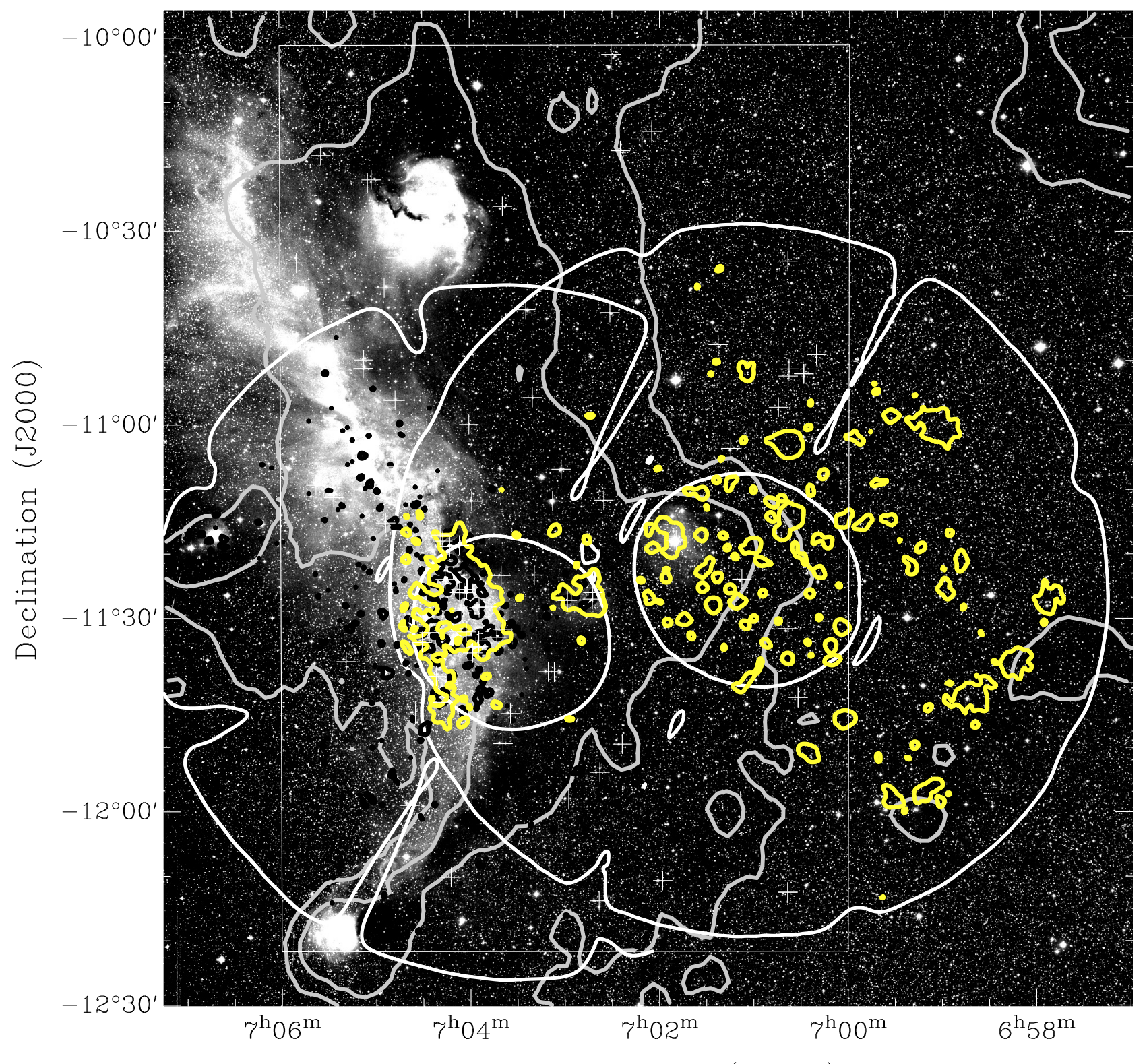

Right Ascension (J2000)

Fig. 2. ROSAT PSPC X-ray contours and fields-of-view superimposed on a digitized POSS(R) image of the CMa R1 region. Grey contours show the same IRAS-ISIS data at $100 \mu \mathrm{m}$ as Fig. 1. The observations of Field 1 are indicated by yellow contours and of Field 2 by black contours. The white crosses indicate the association members identified in the optical survey by Shevchenko et al. (1999), within the area outlined by a white rectangular box.

and has been partially published as part of a survey of Herbig AeBe stars, here Z CMa (Zinnecker \& Preibisch 1994). In such exposures, the number of X-ray counts per source is in general too small to allow deriving a spectrum, or even a good hardness ratio. Therefore, the intrinsic X-ray luminosity of the sources, known to be PMS stars in this case, is based on the count rate, assuming an average plasma temperature (here $k T_{\mathrm{X}} \sim 1 \mathrm{keV}$ ), and is corrected for extinction using the column density given by $N_{\mathrm{H}}=2.1 \times 10^{21} A_{\mathrm{V}} \mathrm{cm}^{-2}$ (e.g., Vuong et al. 2003), $A_{\mathrm{V}}$ being determined by independent methods (see below). A typical value of the approximate X-ray luminosity $\tilde{L}_{X}$ for $A_{V} \sim 1$ is based on the ROSAT count-to-flux ratio $1 \mathrm{ct} \mathrm{ks}{ }^{-1}=9 \times 10^{-15} \mathrm{erg} \mathrm{s}^{-1} \mathrm{~cm}^{-2}$ (see details in Appendix A). The corresponding lower limits to the stellar X-ray luminosities are $\tilde{L}_{\mathrm{X} \text {, min }} \sim 6 \times 10^{29} \mathrm{erg} \mathrm{s}^{-1}$ for Field 1 , and $\tilde{L}_{\mathrm{X}, \min } \sim 1 \times 10^{30} \mathrm{erg} \mathrm{s}^{-1}$ for Field 2 .
Figure 2 is a composite image of Fields 1 and 2, superimposed on a digitized POSS(R) image. The analysis of Field 1 (west of Sh2-296; exposure $\sim 20 \mathrm{ks}$ ) reveals 61 X-ray sources, the majority of them (47) located in the more sensitive central part of the ROSAT field. Field 2 (overlapping Sh2-296; exposure $\sim 5 \mathrm{ks}$ ) contains 37 more sources, which appear much more concentrated in the central region; the outer regions are probably not sufficiently exposed to reveal additional sources. The clustering of Field 2 sources does appear in Field 1, in the form of an extended area of emission, but, being close to the edge of the PSPC FOV, the sources are not resolved individually and thus are not included in the list of Field 1 sources. In contrast, these sources are resolved in Field 2. Five sources are detected in the overlapping area between the two fields. In total, 98 ROSAT sources were detected in these fields. Table A.1 lists 56 distinct sources 


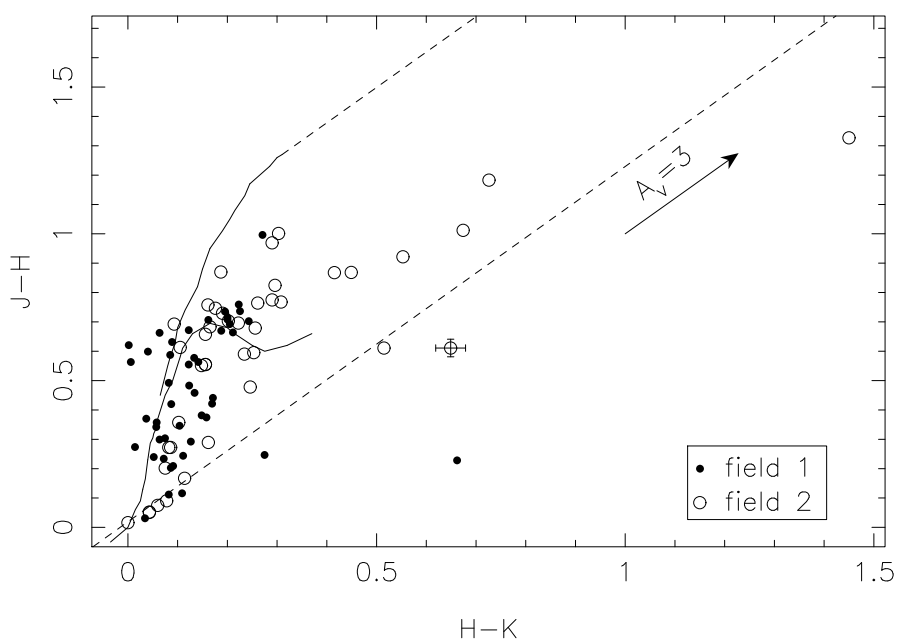

Fig. 3. Diagram $J-H$ vs. $H-K$ of resolved CMaX sources detected in ROSAT fields 1 (filled circles) and 2 (open circles). Full lines indicate the main sequence and red giant branch, and dashed lines are used to show the direction of the interstellar reddening vector. Error bars are shown for a representative $\mathrm{CMaX}$ source.

belonging to Field 1, and 37 belonging to Field 2, and 5 more being detected in both fields. Most of the sources have at least one optical counterpart. Based on their count rates and the estimate of their bolometric luminosities (see next section), we find $\log \left(\tilde{L}_{\mathrm{X}} / L_{\text {bol }}\right)$ lying in the -6 to -4 range, typical of low-mass young stars.

What is equally remarkable is the absence of sources in some areas. In Field 2, the near-absence of sources elsewhere than in the most sensitive central PSPC ring is very likely an observational bias resulting from the short exposure: except perhaps for the brightest, several of the sources visible in Field 1 would not be detected in Field 2 (detection limit: $\log \tilde{L}_{X}=30$ ). In contrast, in Field 1 the absence of sources outside the detected X-ray clustering is very significant. In particular, in the overlapping area between Field 1 and the survey area of Shevchenko et al. (1999, see Fig. 2), no star is detected north and south of GU CMa $\left(\alpha \approx 7^{\mathrm{h}} 02^{\mathrm{m}}\right)$. It is therefore clear that the clustering of $X$ ray sources visible in Field 1 corresponds to a physical cluster, spatially distinct from the B stars identified by Shevchenko et al. (1999). GU CMa certainly belongs to this new cluster, and it is probable that FZ CMa does too, confirming the suspicion of Shevchenko et al. (1999) that these stars may have originated in a different star formation episode. We will address this question in detail in the next sections, adopting the name "GU CMa" for the new cluster.

Appendix A describes the X-ray observations and Table A.1 lists the 98 sources detected in the ROSAT fields, which we name $\mathrm{CMaX}$-(number) for convenience. Two methods were adopted to look for counterparts of the $\mathrm{CMaX}$ sources: (i) using available catalogues (described in Sect. 3), and (ii) in case no counterpart was found, using photometric data from follow-up Gemini observations (see Sect. 4).

\section{Stellar identifications}

From the available images and catalogues listed below, we found at least one optical and/or infrared counterpart for $91 \%$ of the ROSAT sources, 13 of them having known spectral types; the most massive are five B2-3 type stars. Nine X-ray sources coincide with the emission line stars surveyed by Clariá (1974) and/or Schevchenko el al. (1999); their identifications from these catalogues are denoted by "C" and "S" respectively in Table A.1. We note that Z CMa, FZ CMa and GU CMa are detected respectively as CMaX-63, -57, and -48. In addition, nine ROSAT sources coincide with the Soares \& Bica clusters: two in BRC27 (CMaX-74, -75), three in NGC2327 (CMaX-76, -77, -81), and four in vdB-RN92 (CMaX-66, -68, -72, -73). These identifications are also noted in Table A.1.

Optical counterparts for the CMaX sources of Field 1 were first identified on a POSS(R) plate kindly digitized for us by the MAMA $^{1}$ device. However the corresponding MAMA data are not available for Field 2, so for consistency we use the USNO catalogue for the identification and $\mathrm{R}$ magnitudes of the optical counterparts in both fields. The 2MASS catalogue was also used to obtain the IR photometry of the stellar counterparts in the JHK bands. The adopted positional accuracy of the optical counterparts is based on the brightest star located inside the ROSAT error circle. Table A.1 gives these errors, which are less than 10 arcsec for sources in the central areas, and 30 arcsec at the edge of the fields.

We have also used the XMM-Newton and Chandra data, when available, to check the consistency of the positional accuracy of the optical counterparts to the $\mathrm{CMaX}$ sources located in the center area of Field 2, which corresponds to the best ROSAT resolution. Sixteen CMaX sources (listed in Table A.2) have been detected by XMM-Newton and Chandra in this area, confirming the identifications based on ROSAT positions.

Figure 3 shows the $J-H$ vs. $H-K$ diagram obtained for the near-IR counterparts. This diagram indicates that most CMaX sources do not suffer high extinction. Those above the main sequence are located in the direction of the reddening lines, allowing a reliable extinction correction. As explained below (Sect. 4.3), the reddening was estimated from the visual extinction derived from star counts, and checked using two control regions containing field stars. However, four objects present a real $H-K$ excess (appearing to the right side of the dashed line in Fig. 3), which is an indicator of the presence of circumstellar matter. Among them, we find the well-known Herbig star $\mathrm{Z}$ CMa, which has $H-K \sim 1.5 \mathrm{mag}$. There are also some of the X-ray fields for which we could not find any counterpart. In that case, the position of the X-ray centroid is indicated between parentheses in Table A.1. Since these sources have X-ray luminosities in the range $\log \left(\tilde{L}_{\mathrm{X}}\right) \sim 29.4-30.4$, one would expect a counterpart brighter than $R \sim 16$, based on the abovementioned $\left(L_{\mathrm{X}}, M_{R}\right)$ correlation holding for young low-mass stars (further discussed in Sect. 4.4). In a search for counterparts fainter than the POSS(R) plate or USNO limit ( $R \sim 19 \mathrm{mag}$ ), we have obtained deeper observations in the $V, R$ and $I$ bands with the Gemini South telescope. Combining them with the 2MASS catalogue, we obtain altogether VRIJHK photometry for the detected counterparts. The search for these additional, faint counterparts to the unresolved ROSAT sources, which we name the "Gemini candidates", is discussed in the next Section.

\section{Search for faint counterparts}

\subsection{Gemini VRI images and optical magnitudes}

Five fields were observed with Gemini, searching for optical counterparts to X-ray contours for which the centre is "empty".

Their positions, all within the ROSAT Field 1, are shown in Fig. 4. We use the correlations between X-ray luminosities

\footnotetext{
1 Machine Automatique à Mesurer pour l'Astronomie, Observatoire de Paris; now discontinued operation.
} 


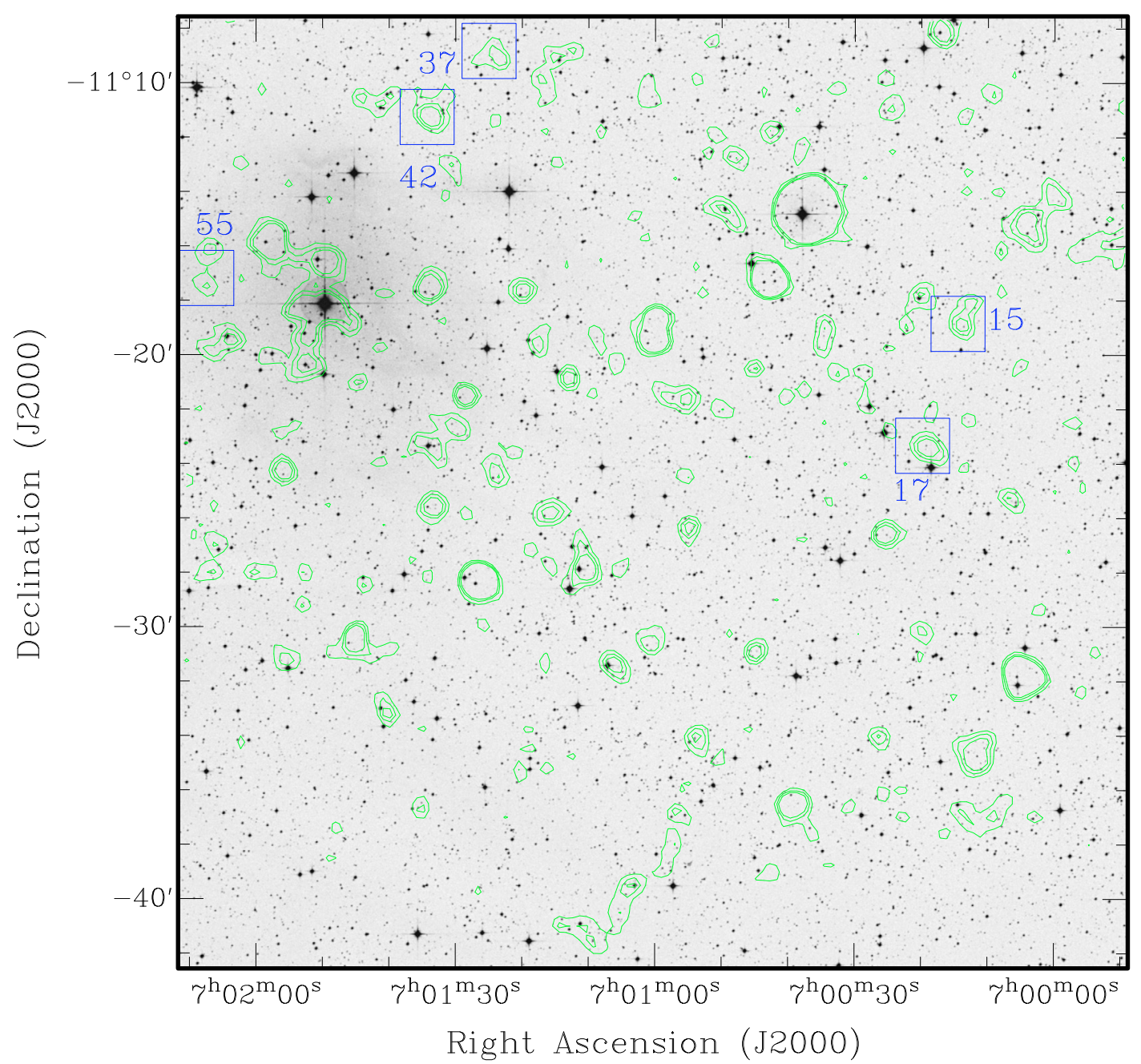

Fig. 4. X-ray sources (green contours) and observed Gemini fields (blue squares, labelled by the corresponding CMaX numbers), superimposed on the POSS (R) image. The X-ray contours were extracted from the ROSAT image using a block bin factor of 30 (see Sect. 4.4), smoothed using a Gaussian PSF with $\sigma=2$ pixels.

and absolute magnitudes $\left(\log \left(\tilde{L}_{\mathrm{X}}\right)\right.$ vs. $M_{R}$ and $\log \left(\tilde{L}_{\mathrm{X}}\right)$ vs. $\left.M_{J}\right)$, obtained for young low-mass stars and displayed in Figs. A.1 and A.2. These correlations show $1 \sigma$ deviations of $1.4 \mathrm{mag}$ in $R$ band and $1.2 \mathrm{mag}$ in $J$. We estimated the expected apparent $R$ and $J$ magnitudes of these counterparts, using a distance modulus of $10 \mathrm{mag}$, with the result that these magnitudes are well above the POSS(R) and 2MASS limits. Yet the counterparts to such bright X-ray sources do not have corresponding optical magnitudes: CMaX15, for example, should be associated with an object having $R=(13.2 \pm 1.4)$ mag, but the brightest object within the $\mathrm{X}$-ray contours has $R=17.1$. These numbers are consistent with the published USNO accuracy ( $\sigma= \pm 0.3 \mathrm{mag}$ ). The $L_{\mathrm{X}} / M_{R}$ ratio in this case is 3.9 orders of magnitude higher than the average value observed in T Tauri stars, i.e., $2.8 \sigma_{R}$ away from the correlation. This fact motivated us to search for multiple faint counterparts that could correspond to unresolved X-ray emitters.

Table 1 gives coordinates of the centroid of the target ROSAT sources, their X-ray properties, and their expected $R$ and $J$ apparent magnitudes. It also gives the number of candidate counterparts for each field, as explained in Sect. 4.2, and their extinction, detailed in Sect. 4.3.

The observations were done with the Acquisition Camera at the $8.1 \mathrm{~m}$ Gemini South telescope, which provides $\sim 2^{\prime} \times 2^{\prime} \mathrm{im}$ ages with a resolution of $0.12 \mathrm{arcsec} / \mathrm{pixel}$. We used the $V$ $\left(\lambda_{\mathrm{c}}=540 \mathrm{~nm}\right), R\left(\lambda_{\mathrm{c}}=640 \mathrm{~nm}\right)$ and $I\left(\lambda_{\mathrm{c}}=790 \mathrm{~nm}\right)$ filters, with total exposure times of $360 \mathrm{~s}$ for the $V$ images, and $300 \mathrm{~s}$ for $R$ and $I$. The fields containing sources CMaX-15, 37, and 55 were observed in 2001, October (Program ID: GS-2001BQ41), while CMaX-17 and 42 were observed in 2003, February (Program ID:GS-2003A-Q06). This last run had weather conditions better than those of the first run. The usual data reduction has been made using bias, flat-field, and dark corrected images provided by the Gemini Observatory.

The photometric calibration of the images was performed using common objects in the USNO B1.0 catalogue (Monet et al. 2003) for the $I$ filter and in the NOMAD catalogue (http://www. navy.mil/nomad.html) for the $V$ and $R$ filters. The number of objects used in the calibration is typically 20 in $R$ and $I$, and 5 in $V$. The brighter objects, which are saturated in the Gemini images, have not been used in the calibration procedure. We have transformed the USNO R magnitude into the Landolt system using the expression given by Kidger (2003). No colour correction was applied.

Figure 5 shows a comparison between the magnitudes in the NOMAD and USNO catalogues and those obtained using our calibration (Monet et al. 2003). We have measured the magnitudes of over a hundred stars in each field. The RMSs of these differences are: $0.26(V), 0.44(R)$ and $0.35(I)$. These numbers are consistent with the published USNO accuracy ( $\pm 0.3 \mathrm{mag})$. 
Table 1. List of CMaX sources observed with Gemini.

\begin{tabular}{|c|c|c|c|c|c|c|c|c|c|c|c|}
\hline \multirow[b]{2}{*}{$\mathrm{CMaX}$} & \multicolumn{6}{|c|}{ X-ray source } & \multicolumn{2}{|c|}{ Expected $^{a}$} & \multicolumn{2}{|c|}{ Candidates $^{b}$} & \multirow[b]{2}{*}{$\begin{array}{c}A_{\mathrm{V}} \\
(\mathrm{mag})\end{array}$} \\
\hline & $\begin{array}{c}\text { RA } \\
\text { J2000 }\end{array}$ & $\begin{array}{c}\text { Dec } \\
\text { J2000 }\end{array}$ & $\begin{array}{l}\text { Err } \\
\left({ }^{\prime \prime}\right)\end{array}$ & Cnts/ks & $\mathrm{S} / \mathrm{N}$ & $\begin{array}{l}\log L_{X} \\
\text { (erg/s) }\end{array}$ & $\begin{array}{c}J \\
(\mathrm{mag})\end{array}$ & $\begin{array}{c}R \\
(\mathrm{mag})\end{array}$ & Optical & Near-IR & \\
\hline 15 & $07: 00: 15$ & $-11: 18: 51$ & 10 & 1.9 & 4.8 & 30.39 & 11.8 & 13.3 & 12 & 6 & $1.23 \pm 0.03$ \\
\hline $17 \mathrm{~N}$ & 07:00:19 & $-11: 22: 10$ & 9 & 0.38 & 1.7 & 29.69 & 14.0 & 16.5 & 4 & 4 & $0.6 \pm 0.3$ \\
\hline $17 \mathrm{~S}$ & 07:00:20 & $-11: 23: 20$ & 9 & 1.31 & 4.3 & 30.23 & 12.3 & 14.0 & 12 & 5 & $0.6 \pm 0.3$ \\
\hline 37 & $07: 01: 25$ & $-11: 08: 50$ & 15 & 2.1 & 5.3 & 30.44 & 11.7 & 13.1 & 15 & 5 & $0.4 \pm 0.6$ \\
\hline 42 & 07:01:34 & $-11: 11: 16$ & 10 & 1.8 & 4.5 & 30.37 & 11.9 & 13.3 & 32 & 9 & $0.4 \pm 0.6$ \\
\hline $55 \mathrm{~S}$ & 07:02:07 & $-11: 17: 11$ & 30 & 0.59 & 2.0 & 29.88 & 13.4 & 15.6 & 5 & 3 & $1.2 \pm 1.0$ \\
\hline $55 \mathrm{~N}$ & 07:02:08 & $-11: 16: 15$ & 30 & 0.72 & 2.6 & 29.97 & 13.1 & 15.2 & 2 & 1 & $1.2 \pm 1.0$ \\
\hline
\end{tabular}

Columns description: (1) CMaX number; $(2,3)$ coordinates; (4) diameter of the position error circle; $(5,6)$ count rate and respective signal-to-noise ratio; (7) X-ray luminosity; $(8,9)$ Statistically expected near-IR and optical magnitudes calculated by adopting the correlation $\log L_{X} /$ absolute magnitude found for young stars (see Sect. 4.4); $(10,11)$ Number of possible optical counterparts within the X-ray contours, and number of corresponding objects found in the 2MASS catalogue; (12) adopted visual extinction based on a standard reddening law.

Notes: (a) The estimated errors are $\sigma_{J}=1.2$ mag. and $\sigma_{R}=1.4$ mag.; (b) (less than $50 \%$ of the candidates have near-IR counterpart).

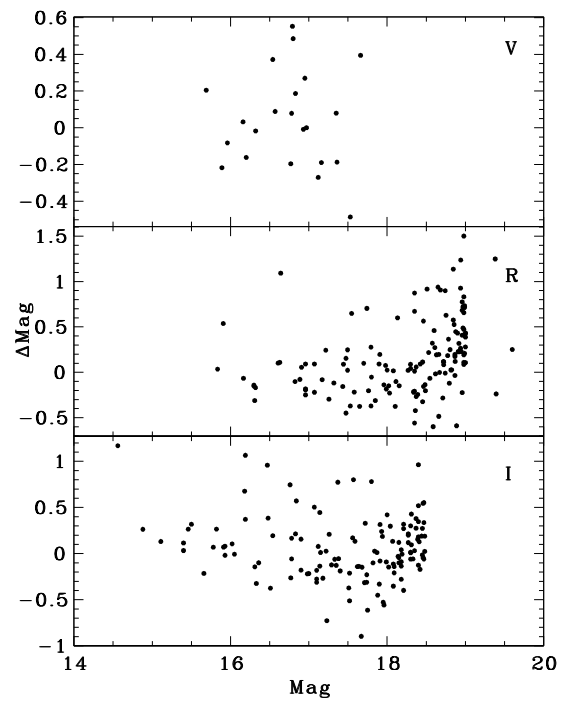

Fig. 5. Difference between our calibrated and catalogue magnitudes as a function of catalogue magnitude for objects in our Gemini fields. From top to bottom, the panels give the $V, R$, and $I$ measurements.

\subsection{Finding candidate counterparts}

The nominal angular resolution of the standard ROSAT image shown in Fig. 2 is 30 arcsec/pixel. To bring out spatial details in X-rays, we extracted images having an enhanced resolution ( $5 \mathrm{arcsec} / \mathrm{pixel})$ for each of the Gemini fields, by adopting a blocking factor of 10 . In this way we can map X-ray contours, including sub-structures, in order to look for unresolved optical candidates that may contribute to the X-ray emission.

The resulting X-ray maps, superimposed on the Gemini I-band images, are given in Fig. 6 (as examples) and in Appendix B (for completeness). Two sources, CMaX-17 and CMaX-55, can be broken down into distinct sub-structures, for which the count rate was then integrated within the X-ray contours, using the same count-to-flux ratio as used for the original sources themselves (see above, Sect. 2). We name them CMaX$17 \mathrm{~S}$ (south) and $17 \mathrm{~N}$ (north), and similarly CMaX-55S and 55N. For three other sources, CMaX-15, CMaX-37, and CMaX-42, no sub-structure is statistically significant.

Figure 6 illustrates two different cases, $\mathrm{CMaX}-15$ and CMaX-17(N+S). Comparing the X-ray contours and the spatial distribution of the stars, we find that many (up to 30) relatively bright objects fall within the X-ray contours, indicating that one or more of these objects contributes to the X-ray emission. Conservatively, we first consider them all as candidate
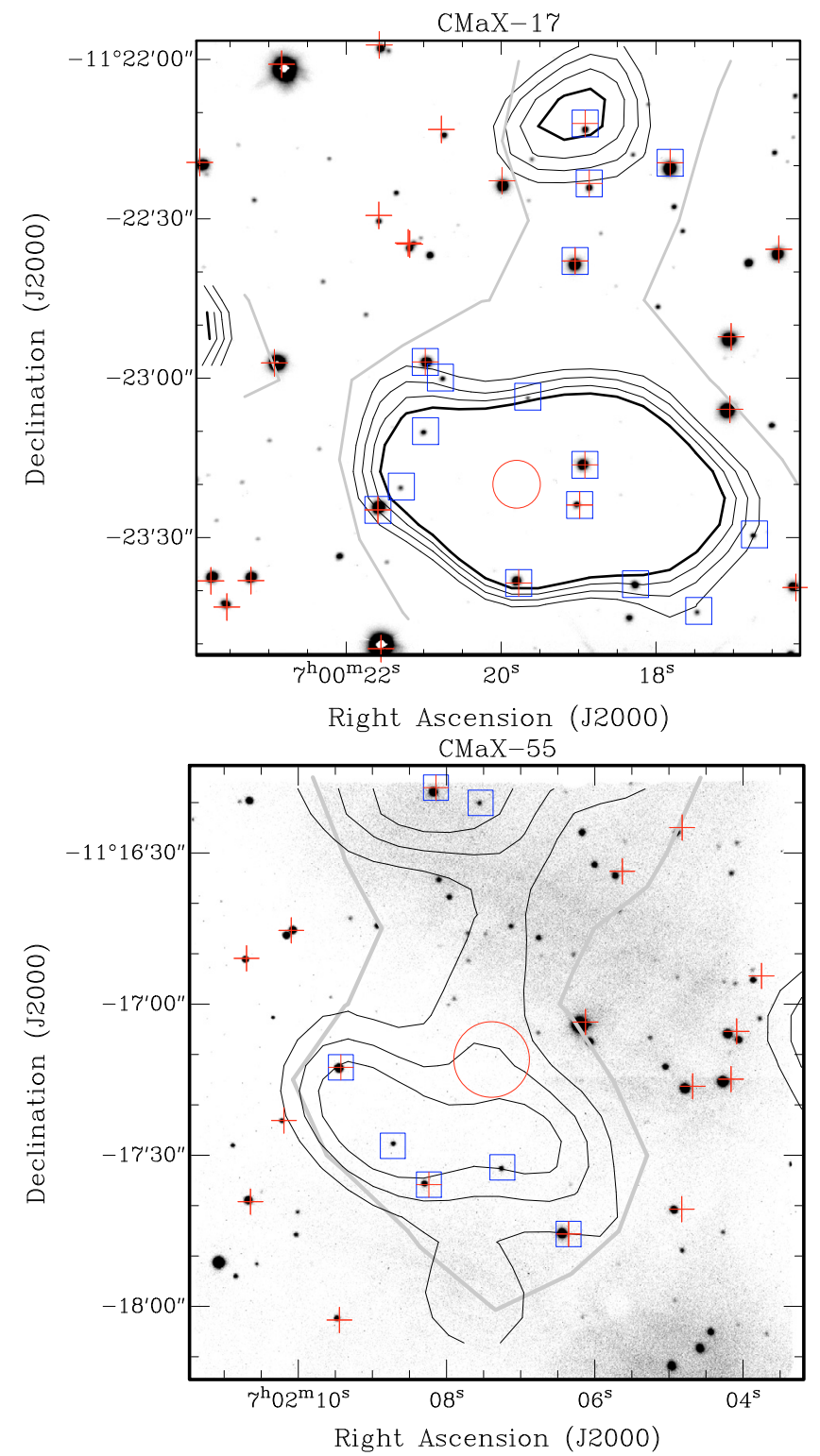

Fig. 6. Optical image obtained by Gemini (I band) of CMaX-17 (top) and CMaX-55 (bottom), two examples of "extended" CMaX sources. Black contours show the X-ray map having enhanced resolution (5 arcsec/pixel), while the nominal resolution (30 arcsec/pixel) is illustrated by the grey line. A red circle indicates the ROSAT nominal position error; red crosses show the 2MASS sources present in the Gemini field, while blue squares show the putative candidates. 
counterparts to the unresolved ROSAT sources. Other objects are also visible on the optical image, but they are fainter and no reliable Gemini photometry could be obtained for them. Therefore, we restrict our analysis to objects brighter than $R=21 \mathrm{mag}$.

To further characterize candidate counterparts, we searched for near-IR sources within the Gemini fields using the 2MASS catalogue. These sources are shown by crosses in Fig. 6, while the relevant optical-near-IR identifications (i.e., within the X-ray contours) are indicated by open squares. More than half of the visible objects within the X-ray contours are too faint to appear in the 2MASS catalogue (limiting magnitude $J=17$ ). As a consequence, as shown in Table 1 , only a fraction $(\sim 46 \%)$ of the candidates have VRIJHK data (6 of 12 for CMaX-15, 9 of 16 for CMaX-17, etc.).

We now have a first list of optical candidates selected on the basis of position (within the X-ray contours) and brightness $(R<$ $21)$. But this list may still include foreground and background objects, and we have to find their intrinsic colours (using VRI photometry from Gemini, and $J H K$ photometry from 2MASS when available), to establish their young-star nature, and to do so we must now consider interstellar extinction and IR excess (possibly indicative of circumstellar disks).

\subsection{Interstellar reddening}

The large-scale extinction models of Amôres \& Lépine (2005), which are based on gas ( $\mathrm{HI}$ and $\mathrm{CO}$ ) and dust (IRAS) column densities, have a $\sim 0.5^{\circ}$ spatial resolution. They give $A_{V}=$ $0.4 \mathrm{mag}$ for the foreground extinction in the direction of $\mathrm{CMa}$ $\mathrm{R} 1$. This is however a lower limit on a smaller spatial scale, since the presence of CMa cloud must be also taken in account. We have thus estimated the extinction towards the ROSAT sources using star counts.

First we used the digitized POSS(R) image of the entire ROSAT field with a resolution of $2.5^{\prime}$ (Sect. 3). More accurate estimates were kindly provided by Cambrésy (2002, private communication), who obtained an extinction map of the CMa R1 region based on source counts in the $J$ band, from the near-IR DENIS catalogue. This method is the same as that used for the North America and Pelican nebulae (Cambrésy et al. 2002).

Aiming to obtain the extinction in both optical (VRI) and near-IR (JHK) bands, the conversion of $A_{\lambda} / A_{\mathrm{V}}$ was done by adopting the reddening law from Cardelli et al. (1989) and the reddening-to-selective extinction ratio $R_{\mathrm{V}}=4$, which Terranegra et al. (1994) estimate in the direction of CMa R1. Last column of Table 1 gives the adopted visual extinctions and deviations of these values, measured in each Gemini field.

In order to check whether the magnitudes were correctly dereddened, field stars were selected in two control regions. A first region considers stars in a local area $\left(1.5^{\prime}\right.$ radius around the X-ray source centroid), indicated by crosses in Fig. 6 (excluding the candidates). The colours of the stars in this area were corrected by using the same extinction as adopted for the candidates. The other region is located around $\alpha_{(\mathrm{J} 2000)} \sim 07^{\mathrm{h}}$, $\delta_{(\mathrm{J} 2000)} \sim-10^{\circ}$ (upper right-hand corner of Fig. 1), which is beyond the lowest IRAS contour and thus is free of cloud contamination. In this case, the stars were de-reddened by using the foreground extinction $A_{J}=0.12 \mathrm{mag}$ (equivalent to the value $A_{V}=0.4$ mentioned previously from Amôres \& Lépine (2005)). Figure 7 displays the $(J-H)_{0}$ vs. $(H-K)_{0}$ diagram (de-reddened near-IR colours) for the objects studied in the Gemini fields. The intrinsic colours of the ZAMS stars (Siess et al. 2000) and giants (Bessell et al. 1998) are also plotted. The comparison with field stars of the control regions indicates that the extinction

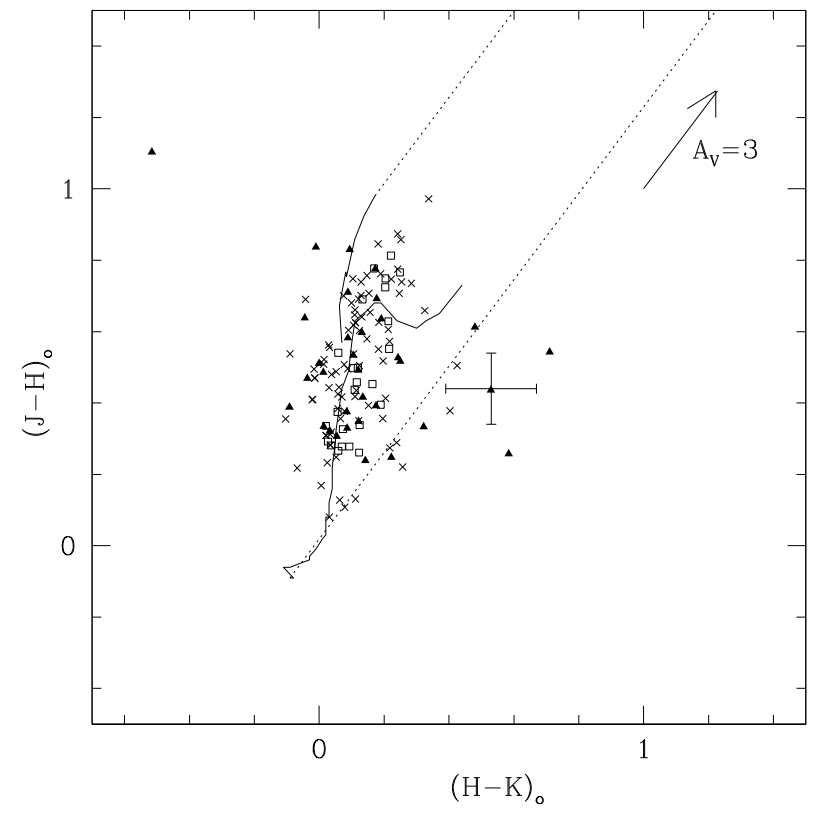

Fig. 7. Near-IR colour-colur diagram of the possible counterparts of the X-ray sources studied in the Gemini fields (triangles). A typical error bar is shown for illustration. Two control groups of field stars are indicated by: (i) crosses for objects near $(\sim 1 \mathrm{arcmin})$ to the centre of the Gemini image; and (ii) open squares corresponding to stars located in a distant region free from obscuration. The full lines represent the zero age main sequence and giant intrinsic colours. Interstellar reddening vectors are indicated by dashed line for B8V and M7III stars.

corrections given in the last column of Table 1, can be applied to the optical magnitudes of the Gemini candidates.

\subsection{Confirmed candidates}

The intrinsic colours of the Gemini candidates were then analyzed for two different purposes: (i) near-IR colour-colour diagrams, looking for H-K excesses; (ii) optical colour-magnitude diagrams, to confirm their young star nature and determine their masses and ages.

It can be noted in Figs. 7 and B.2 (Appendix B) that three counterparts in Gemini fields show $H-K$ excess. Even considering deviations on the corrections for extinction, the $H-K$ excess is still high for these objects. This indicates the presence of circumstellar matter, as suggested for other four objects in Fields 1 and 2 (Sect. 3). The relevant sources are indicated by an asterisk in Table A.1.

The discussion of the optical characteristics of the counterparts in Gemini fields is based on the colour-magnitude diagram, using the $R$ and $I$ bands, and a distance modulus of 10 mag. Figure 8 displays the absolute red magnitude $\left(M_{R o}\right)$ as a function of $(R-I)_{0}$ (both corrected for extinction), compared to the theoretical models calculated for the ZAMS, $10 \mathrm{Myr}, 1 \mathrm{Myr}$, and $0.1 \mathrm{Myr}$, in the mass range from 0.1 to $7 M_{\odot}$ (Siess et al. 2000).

Optical colours (Fig. 8) and near-IR colours (Fig. 7) were both used to select the counterparts, based on colour excess. Therefore the objects having colours similar to the Field stars (located in the left side of the ZAMS) were disregarded, while the objects showing $(H-K)$ or $(R-I)$ excess (appearing above the ZAMS) were kept as counterparts. Adding this constraint, we can establish a final list of confirmed young star counterparts, which is given in Table 2. Among the selected counterparts, CMaX42-1 is the only one that is not associated to a nearIR source. In order to have a homogeneous sample of objects, 


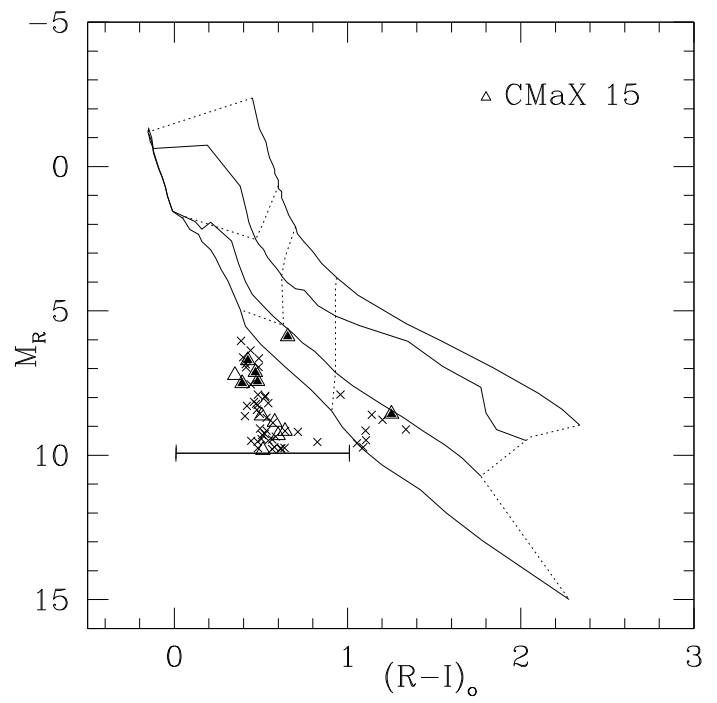

Fig. 8. Colour-magnitude diagram of the optical candidates to be counterparts of the X-ray source CMaX15. $R$ - and $I$-band data were extracted from Gemini images and corrected for reddening using the extinction estimated for each field (see text). An error bar typical of faint objects is shown for illustration. The field stars are indicated by crosses, while candidates are shown by triangles (filled symbols represent near-IR sources). The theoretical lines (intrinsic colours and isochrones) were taken from Siess et al. (2000). The isochrones $0.1 \mathrm{Myr}, 1 \mathrm{Myr}$, and $10 \mathrm{Myr}$, and the zero age main sequence (full lines) and evolutionary tracks for $0.1,0.5,1,2$, and $7 M_{\odot}$ (dotted) are plotted. The absolute $M_{R}$ magnitude was obtained by adopting a distance modulus of $10 \mathrm{mag}$.

we decided to exclude this object of the overall analysis. This exclusion does not affect our results, since it is the faintest counterpart of CMaX42, giving a small contribution to the total X-ray luminosity, as explained below.

The unresolved ROSAT sources in Field 1 are thus found to have up to two or four plausible counterparts each. There are two extreme possibilities: (i) all the counterparts contribute more or less equally to the X-ray luminosity, or (ii) only one dominates the $\mathrm{X}$-ray emission.

To do so, we compare the values of $L_{\mathrm{X}}$ of the original ROSAT sources, with the "integrated" $J$ and $R$ magnitudes of the confirmed counterparts, obtained by summing the individual $J$ and $R$ luminosities (derived from the individual $J$ and $R$ magnitudes), and converting back the result into magnitudes. For a given CMaX source, we list in Table 2 the two extreme magnitudes (mentioned above): the "integrated" magnitudes $R_{\text {int }}$ and $J_{\text {int }}$, and the individual magnitudes of the brightest candidate.

In Appendix A we compare the integrated magnitudes to the X-ray luminosity. More precisely, we show in Figs. A.1 and A.2 a diagram of $L_{X}$ versus $M_{R o}$ and $M_{J o}$. The Gemini "integrated" candidates are found to lie on the high side, within $\sim 2 \sigma$ of the nominal correlation. This suggests that the X-rays are dominated by a small number of candidates (which would diminish the absolute magnitudes for a given X-ray luminosity). But since the evidence is not clear-cut, we will conservatively assume that the $\mathrm{X}$-ray emission is spread among the candidates, in other words that all the candidates listed in Table 2 (excepting CMaX42-1) are X-ray emitting young stars, to be added to the list of the resolved CMaX sources.

\section{Stellar masses and ages}

The stellar masses and ages of the resolved CMaX sources (Table A.1), and of the faint Gemini counterparts for the

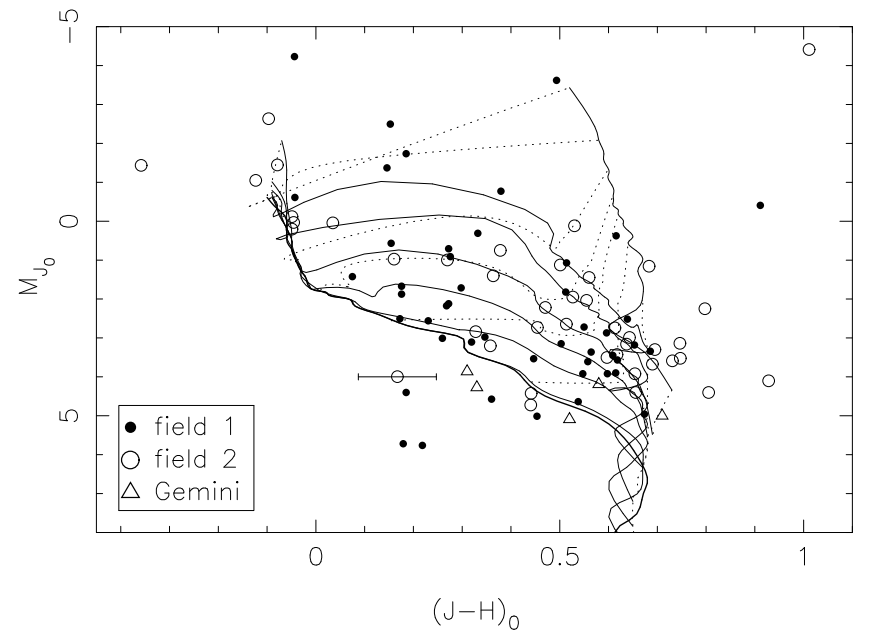

Fig. 9. Diagram of absolute $J$ magnitude as a function of the $J-H$ colour (both corrected for extinction). The CMaX sources in Field 1 are represented by filled circles, sources in Field 2 are shown by open circles. Five representative Gemini sources are shown by triangles. The zero age main sequence (ZAMS) and isochrones ( 0.1 to $20 \mathrm{Myr})$ are indicated by full lines and evolutionary tracks $\left(0.1\right.$ to $\left.7 M_{\odot}\right)$ by dotted lines.

unresolved sources, were derived from different colourmagnitudes diagrams, and compared with the pre-main sequence evolutionary models of Siess et al. (2000). For the CMaX sources we used the near-IR $M_{J}$ vs. $J-H$ diagram (Fig. 9). For the Gemini candidates we used the $M_{R}$ vs. $R-I$ diagrams (Figs. 8 and B.3), since magnitudes are too faint, making a comparison difficult with the isochrones in the near-IR colour-magnitude diagram. Only near-IR isochrones are used in the comparison of age distribution of CMaX resolved sources in Fields 1 and 2.

From Fig. 9, we find that 18 resolved $\mathrm{CMaX}$ sources are located "outside" the isochrones (adopting error bars of \pm 0.3 mag in $J-H)$, so we could determine masses and ages only for 40 of them in Field 1, and for 31 in Field 2. The error bars of $J-H$ color indicate that errors of $30 \%$ to $50 \%$ can be expected for age and mass. For this reason, to estimate these parameters we decided to adopt ranges (or bins) defined by the isochrones separation (5-10 Myr, for example), instead of nominal values $(7.5 \pm 2.5 \mathrm{Myr}$, for example). These determinations are listed in Table A.1, except for sources marked with "??". Among the 18 objects with undetermined age and mass, 14 are located to the right side of the $0.1 \mathrm{Myr}$ isochrone: this is probably due to the presence of circumstellar matter typical of YSOs. On the other hand, four objects located to the left side of the isochrones are probably not the right counterparts of the CMaX sources. Indeed, other faint objects are found within $30^{\prime \prime}$ of the center of the corresponding X-ray contours, but the lack of photometry for these four objects does not affect the age and mass distribution of CMaX sources.

Considering that counterparts identified in the Gemini fields have $M_{J}>5$ mag., most of them appear outside of the isochrones of the near-IR colour-magnitude diagram. Thus, Fig. 9 does not show the distribution of all the Gemini counterparts, but, for illustration, only of the five brightest sources per field. In this case, optical colours are more appropriate to determine the stellar parameters: their ages and masses were estimated from $M_{R}$ vs. $R-I$ diagrams shown in Figs. 8 and B.2 (Appendix B). From Fig. 9, one object (CMaX15-1) is approximately $20 \mathrm{Myr}$ old and has a mass $\sim 1 M_{\odot}$, in agreement with the result $\left(10-20 \mathrm{Myr}, 0.5-1.0 M_{\odot}\right)$ obtained from Fig. 8 and indicated in Table 2. 
Table 2. List of possible additional faint counterparts to CMaX sources.

\begin{tabular}{|c|c|c|c|c|c|c|c|c|c|c|c|c|}
\hline CMaX & $\begin{array}{c}\text { RA } \\
\text { J2000 }\end{array}$ & $\begin{array}{c}\text { Dec } \\
\text { J2000 }\end{array}$ & $\begin{array}{c}R_{\text {int }} \\
(\mathrm{mag})\end{array}$ & $\begin{array}{c}R \\
(\mathrm{mag})\end{array}$ & $\begin{array}{l}V-R \\
(\mathrm{mag})\end{array}$ & $\begin{array}{l}R-I \\
(\mathrm{mag})\end{array}$ & $\begin{array}{c}J_{\text {int }} \\
(\mathrm{mag})\end{array}$ & $\begin{array}{c}J \\
(\mathrm{mag})\end{array}$ & $\begin{array}{c}J-H^{d} \\
(\mathrm{mag})\end{array}$ & $\begin{array}{c}H-K^{d} \\
(\mathrm{mag})\end{array}$ & $\begin{array}{c}\text { Age } \\
(\mathrm{Myr})\end{array}$ & $\begin{array}{l}\text { Mass } \\
\left(M_{\odot}\right)\end{array}$ \\
\hline $15-1$ & 70013.364 & -111853.43 & $16.5^{e}$ & $17.1^{a}$ & 0.29 & $1.08^{b}$ & $14.4^{e}$ & 14.77 & $0.70 \pm 0.06$ & $0.19 \pm 0.07$ & $10-20$ & $0.5-1.0$ \\
\hline & & & & & & & & & & & & $? ?$ \\
\hline $15-3$ & & & & 18. & & & & & & & $? ?$ & $? ?$ \\
\hline $15-4$ & 70015.378 & -11193.78 & & 19.7 & 1.6 & $1.56^{b}$ & & 16.45 & $0.76 \pm$ & $0.29 \pm 0.24$ & $10-20$ & $0.1-0.5$ \\
\hline $17 \mathrm{~N}$ & & & & 18.8 & 0.89 & & & & & & & $? ?$ \\
\hline $17 \mathrm{~s}$ & & & $16.9^{e}$ & 17. & 0. & $0.79^{b}$ & $15.4^{e}$ & & & & $20-50$ & $0.5-1.0$ \\
\hline $17 \mathrm{~S} 2$ & 70018.886 & -112323.67 & & 19.2 & 1.22 & $1.15^{b}$ & & 16.71 & 0.89 & 0.29 & $20-50$ & $0.1-0.5$ \\
\hline $37-1$ & 70122.297 & -11099.29 & & 17.7 & 0.9 & $0.97^{b}$ & & 16.18 & $0.65 \pm 0.15$ & $-0.1 \pm 0.24$ & $10-20$ & $0.5-1.0$ \\
\hline $37-$ & & & & 18 & & $1.67^{b}$ & & & & & $1-$ & $0.5-1.0$ \\
\hline $37-3$ & 70124.356 & -110903.00 & $15.9^{e}$ & $16.3^{a}$ & -0.3 & $0.88^{b}$ & $14.3^{e}$ & & $0.55=$ & $0.11 \pm 0.08$ & $5-10$ & $0.5-1.0$ \\
\hline $42-1$ & & & & & & & & & & & $20-50$ & $0.5-1.0$ \\
\hline $42-$ & & & $15.6^{e}$ & $15.7^{a}$ & -0.08 & $0.86^{b}$ & $13.9^{e}$ & & $0.35 \pm 0.06$ & $0.08 \pm 0.08$ & $2-5$ & $0.5-1.0$ \\
\hline $42-3$ & 70135.473 & -111014.46 & & 16.4 & 0.74 & $0.71^{b}$ & & 14.70 & $0.73=$ & $0.21 \pm 0.08$ & $10-20$ & $0.5-1.0$ \\
\hline $55 \mathrm{~N}$ & 7028.021 & -111617.65 & $18.2^{e}$ & $18.2^{a}$ & 0.98 & $1.08^{b}$ & $15.6^{e}$ & & & 0.35 & $10-20$ & $0.1-0.5$ \\
\hline $55 \mathrm{~S}$ & 7028.139 & -111735.46 & & 18.9 & 0.6 & 0.74 & & 16.46 & $0.73 \pm 0.2$ & $0.58 \pm 0.22^{c}$ & $? ?$ & $? ?$ \\
\hline
\end{tabular}

Notes: (a) Magnitudes adopted from the NOMAD and/or USNO catalogues, when the estimation from Gemini images are unavailable, due to saturation; (b) counterparts selected according to the $R-I$ excess; (c) counterparts having $H-K$ excess; (d) the 2MASS catalogue was used to obtain the JHK magnitudes, for which error bars are provided; (e) $R_{\text {int }}$ and $J_{\text {int }}$ represent the "integrated" magnitudes (see Sect. 4.2), which are shown together with the brightest object of each group.
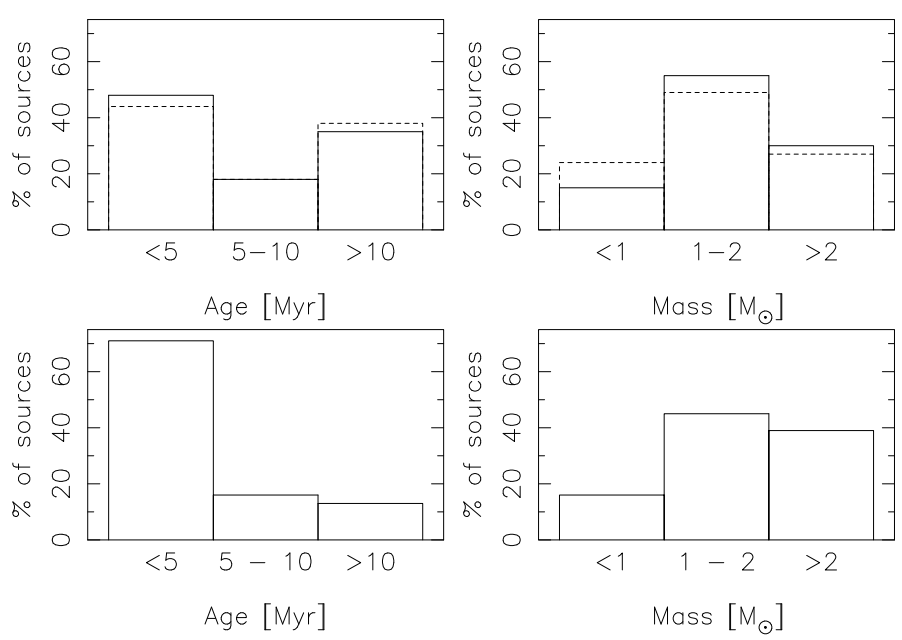

Fig. 10. Age and Mass distribution of the studied sample in Field 1 (top) and Field 2 (bottom). Dashed lines are used to show the contribution of additional counterparts in Field 1, detected by Gemini.

We have compared our results with the cluster ages estimated by Soares \& Bica (2002, 2003; see above, Sects. 1 and 3). Soares \& Bica (2002) give an age of $~ 1.5 \mathrm{Myr}$ for NGC 2327 and BRC 27. Five resolved CMaX sources belong to these clusters, for which we have obtained similar ages. For the older cluster VdBRN92 (5-7 Myr, Soares \& Bica 2003) we have identified four other CMaX sources, but only one of them (CMaX-68) has compatible age, while the others seem to be massive stars for which the age determination is uncertain.

Figure 10 presents the results, in the form of two panels, showing the broad distributions of ages and masses for all the $\mathrm{CMaX}$ sources in Field 1 and Field 2 respectively. The percentage of sources for the each bin was obtained by dividing the number of objects in the bin by the total number of sources in each field.

As concerns the Gemini candidates, two possibilities are explored in the histograms (for Field 1; see Table 2), considering: (i) only the 40 counterparts of resolved CMaX sources (indicated by full lines in Fig. 10); (ii) the brightest Gemini stars as counterparts to unresolved CMaX sources, leading to $40+5$ objects (dashed lines in Fig. 10). Note that objects outside the isochrones of Fig. 9 have not been included in the distributions of ages and masses.

We comment on these results in the next section.

\section{Conclusions}

\subsection{Summary of results}

One way to elucidate the nature of the ionized "CMa R1 ring" or "Arc" (Sh2-296) is to investigate the stellar population in its vicinity, more precisely to reconstruct the star formation history of the region. Until the present work, only the bright-star end of the population (from early $\mathrm{B}$ stars to $\mathrm{F}$ and $\mathrm{K}$ giants), i.e., the high-mass/young population, was surveyed. In the present paper, we used the most powerful tool to find the low-mass, older late-type population, namely their X-ray emission resulting from magnetic activity. To this end, we analyzed or reanalyzed archival ROSAT data.

Two overlapping PSPC fields exist, which cover a wide area, over nearly 5 sq. deg., i.e., the equivalent of a mosaic of 20 $X M M$-Newton fields, or 80 Chandra fields. The price to pay, however, compared to these satellites, is a relatively modest sensitivity, especially in one of the two fields which has a short exposure. More precisely, we find that our ROSAT survey is limited to $M_{\star} \sim 0.5 M_{\odot}$, as opposed to the usual limit of $M_{\star} \sim 0.1 M_{\odot}$ easily reached for typical XMM-Newton or Chandra observations of star-forming regions.

The two ROSAT fields (see Figs. 1 and 2), labelled by order of increasing Right Ascension, are "Field 1", west of the CMa ring (20 ks exposure), and "Field 2", centred on the Herbig star Z CMa and overlapping most of the ring but with a short exposure $(5 \mathrm{ks})$. Fifty-six sources are detected in Field 1, and 37 in Field 2, 5 more being detected in the overlap area between Field 1 and Field 2. Note that, since the exposure is 4 times shorter in Field 2 than in Field 1, the expected number of sources, if similar in nature, would be 2 times less, i.e., 28: therefore, X-ray sources seem more numerous in Field 2 relative to 

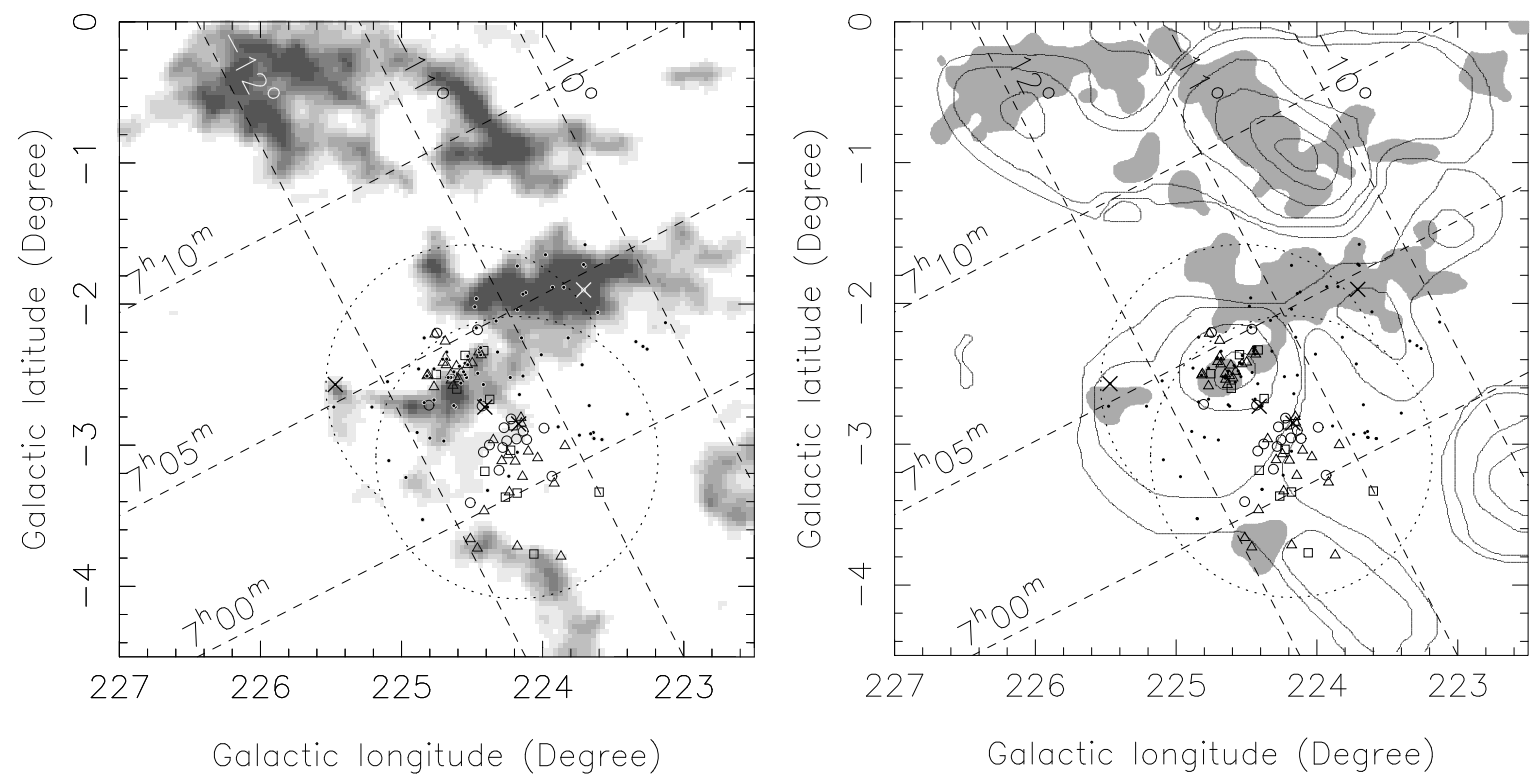

Fig. 11. Spatial distribution of the CMaX sources, in galactic coordinates, compared to: (left) visual extinction (Dobashi et al. 2005) and (right) $\mathrm{CO}$ maps: ${ }^{13} \mathrm{CO}$ is indicated by grey areas and ${ }^{12} \mathrm{CO}$ by full line contours. The $2^{\circ}$ circles (dotted lines) show the ROSAT fields. The different age bins of Fig. 10 are represented by triangles $(<5 \mathrm{Myr}$ ), squares $(5-10 \mathrm{Myr})$, and circles ( $>10 \mathrm{Myr})$. The position of emission line stars detected by Shevchenko et al. (1999) is shown by dots. Large crosses indicate Sh-292, GU CMa, FZ CMa, Sh-297 (ordered by increasing galactic longitude).

Field 1, although the difference is marginally within Poissonian errors. In all, $\sim 100$ sources are detected.

To identify the ROSAT sources, we searched catalogues (USNO and 2MASS), and digitized plates (POSS(R)), in order to characterize them via their colours. Not too surprisingly, most of the X-ray sources have no known counterpart, and we used our own photometry from the POSS(R) plate (Field 1), or USNO magnitudes when unavailable (Field 2), supplemented by 2 MASS data. For five sources having no counterpart inside the position error circle, we observed the corresponding fields with Gemini South to find possible fainter counterparts and determine their VRI magnitudes. The spatial distribution of the sources suggests the existence of two distinct X-ray clusters. One is visible in Field 2, closely associated with Z CMa and the CMa ring, that includes previously known optical clusters studied by Soares \& Bica $(2002,2003)$, and which we will name globally the "Z CMa cluster" hereafter; and a new one, discovered in Field 1, west of the CMa ring, which includes the bright star GU CMa (B2V), spatially distinct from the previous one. We name this newly discovered cluster the "GU CMa cluster".

The presence of circumstellar matter is suggested for 7 counterparts that show $H-K$ excess. This excess does not affect the estimation of stellar parameters such as mass and age, which were derived from $M_{J_{o}}$ vs. $(J-H)_{0}$ diagram. Three figures summarize our X-ray source characterization. Figure 9 shows a colour-magnitude diagram for our sample, and PMS evolutionary tracks (Siess et al. 2000). This yields mass and age estimates for the sources, which are summarized in broad bins in Fig. 10 for the two clusters separately: masses $<1,1-2,>2 M_{\odot}$, ages $<5,5-10,>10$ Myr. Figure 11 locates the position of the ROSAT sources with respect to the molecular gas of the region, traced by ${ }^{12} \mathrm{CO}$, and ${ }^{13} \mathrm{CO}$ for the densest regions, compared to the visual extinction map from Dobashi et al. (2005).

\subsection{Discussion}

Although the error bars on masses and ages are large $(\sim 30 \%)$, Fig. 10 shows significant similarities and differences between the $\mathrm{Z} \mathrm{CMa}$ and GU CMa clusters. (i) The mass functions above $1 M_{\odot}$ are similar, with a maximum in the $1-2 M_{\odot}$ range. Below $1 M_{\odot}$, however, our sample is incomplete, essentially because it misses the X-ray faint, lowmass stars. This is exemplified by the Gemini sources in the GU CMa cluster: they are mostly detected as groups, and thus tend to fill the $<1 M_{\odot}$ mass bin. For both clusters, this mass bin should therefore be considered only as indicative of the low-mass, faint population.

(ii) The age distribution is more significant. The GU CMa cluster appears older than the Z CMa cluster: $35-38 \%$ of the GU CMa members (depending on whether or not the Gemini sources are taken into account) are older than $10 \mathrm{Myr}$, whereas nearly $70 \%$ of the Z CMa cluster stars are younger than $5 \mathrm{Myr}$. (Conversely, $\sim 45 \%$ of the GU CMa members are younger than $5 \mathrm{Myr}$, and $13 \%$ of the Z CMa members are older than 10 Myr: we will return to this point below). In both clusters, the intermediate-age population is similar $(\sim 17 \%$ between 5 and $10 \mathrm{Myr})$.

(iii) The most important difference is the space distribution of the X-ray sources, compared with the gas $\left({ }^{13} \mathrm{CO}\right.$ : Kim et al. 2004; ${ }^{12}$ CO, May et al. 1988) and dust (Dobashi et al. 2005) distributions (Fig. 11; see also Figs. 1 and 2). The Z CMa cluster X-ray sources are extremely well correlated with the CMa ring and ${ }^{13} \mathrm{CO}$ emission from dense gas, consistent with the high fraction of these sources being very young. This demonstrates that an active star formation episode is currently going on, likely induced by the ring, especially if the X-ray source number excess in Field 2 over Field 1 noted above is real. This qualitatively confirms previous optical and IR studies, but adds several tens of young low-mass stars to the existing census. In contrast, the GU CMa cluster is located in a "vacuum", comparatively far from the dense ${ }^{13} \mathrm{CO}$ gas, just at the limit of the lower-density ${ }^{12} \mathrm{CO}$ gas. This is also consistent with the older age of this cluster.

We thus have now evidence for two distinct episodes of star formation, as previously suspected by Schevchenko et al. (1999) for a couple of bright stars (GU CMa and FZ CMa): star formation in the $\mathrm{Z}$ CMa region is currently going on, fueled by dense molecular material at the edge of the CMa ring, but, in the 
absence of such material, must have ceased several Myr ago in the GU CMa cluster.

Yet this evidence is not clearcut: why is there a significant fraction $(16 \%)$ of old stars in the $\mathrm{Z}$ CMa region (a previously known result, as recalled in the Introduction, which we confirm and extend on the basis of lower-mass stars)? And conversely, why is there a significant fraction of young stars (44\%) in the GU CMa cluster? A possible answer is a mixing, due to stellar proper motions, of the two clusters: at a distance of $\sim 1 \mathrm{kpc}$, the centroids of the two clusters would be $\sim 17$ pc apart $\left(\sim 1^{\circ}\right.$ on the sky). Mixing could start to be visible if the stars had a proper motion of order a few $\mathrm{km} \mathrm{s}^{-1}$, which is reasonable, provided the centroids themselves would not have moved significantly. The main objection against such an interpretation is the lack of a "well-mixed" population between the two clusters, which appear spatially very distinct. However, there may be some extinction effect: the "intercluster" region is within a low-density, but extended region visible in ${ }^{12} \mathrm{CO}$ (Fig. 11) and in the IRAS data (Fig. 1), possibly yielding column densities high enough to hide faint X-ray sources. More sensitive X-ray observations would be needed to clarify this point.

A related question is that of the connection between the newly discovered GU CMa cluster, and the surrounding molecular clouds. Figure 11 shows that the GU CMa cluster lies in a cavity, roughly symmetrical (with respect to ${ }^{13} \mathrm{CO}$ contours) to the cavity associated with the CMa ring. This could suggest that the GU CMa cluster cavity has been excavated by stellar winds and/or supernovae from the now defunct high-mass end of the cluster stars. No diffuse X-ray emission is visible in our ROSAT PSPC data, but this is not too surprising in view of the sensitivity required to detect this emission (see the example of Orion with XMM-Newton, Güdel et al. 2008). Some diffuse X-ray emission does appear to exist in this region in the $3 / 4 \mathrm{keV}$ ROSAT diffuse background (Snowden et al. 1997), but it is on a large scale and not directly related to the $\mathrm{CMaX}$ region in particular. An interesting test of the stellar wind excavation hypothesis would be to obtain a census of circumstellar disks (in the near-IR range) around the youngest stars of the cluster (i.e., with ages $<5 \mathrm{Myr}$ and noted by triangles in Fig. 11), which, under normal conditions should have still retained their original disk (e.g., Dahm \& Hillenbrand 2007). Indeed, stellar winds in OB associations are known to be able to quickly blow away disks, as is the case, for instance, in the Sco-Cen association (Preibisch \& Zinnecker 1999). Along these lines, we note that this hypothesis is indeed consistent with the very small number of sources with near-IR excess in the GU CMa cluster.

\subsection{Concluding remarks}

By analyzing archival ROSAT data, which give access to the previously unknown low-mass $\left(M_{\star} \sim 0.5 M_{\odot}\right)$, old stellar population, we have shown in this paper that star formation in the CMa $\mathrm{R} 1$ region has been going on for more than $10 \mathrm{Myr}$, but in two separate episodes, each giving rise to a distinct cluster. The older one, which we name the "GU CMa" cluster, has been discovered only by way of X-ray observations, supplemented by our photometric characterization of the X-ray sources. Given its location far from dense molecular material, it is clear that star formation has now ceased in this cluster. The existence of a younger cluster, which is observed around the Herbig star Z CMa, has been known for some time as a result of previous surveys, but our X-ray observations add several tens of low-mass stars to the existing census. A puzzling question remains, which is the presence in both clusters of a small, but significant fraction of young
$(<5 \mathrm{Myr})$ and older ( $>10 \mathrm{Myr})$ stars. Spatial mixing between the two clusters is a possibility, but more sensitive X-ray observations would be needed (to lower the mass limit and/or overcome the extinction) to clarify this point.

At any rate, there does not seem to be any connection between the GU CMa cluster and the CMa ring. The star formation episode that gave rise to GU CMa and its cluster likely predates the ring, perhaps by as much as a few Myr. Only the clusters in the $\mathrm{Z} \mathrm{CMa}$ region appear related to the ring. It is entirely possible that other older clusters like the GU CMa cluster exist in the region, in particular inside the ring, east of $\mathrm{Z} \mathrm{CMa}$. As demonstrated with the discovery of the GU CMa cluster, observations with wide-Field X-ray telescopes like ROSAT are very powerful to detect such clusters, but unfortunately that era is over, and even with a moderate field-of-view like the EPIC camera aboard $X M M$-Newton, a mosaic of many fields is required to map large areas. Until this is done, the origin of the CMa ring will likely remain an unsolved problem.

Acknowledgements. We would like to express our gratitude to A. Hetem Jr. for his kindness preparing some of the figures (composite maps) that improved the presentation of the paper. We are grateful to J. Guibert for help in acquiring the data from the plate digitizing machine MAMA. The authors thank FAPESP for partial financial support (JGH: Procs. No. 2001/09018-2 and No. 2005/00397-1; CVR: Proc 01/12589-1). JGH and TM thank support from USP/COFECUB (Proc. No. 2007.1.435.14.5) and Fapesp/CNRS (Proc. No. 2006/50367-4). This work has made use of the SIMBAD, VizieR, and Aladin databases operated at CDS, Strasbourg, France. This publication makes use of data products from the Two Micron All Sky Survey, which is a joint project of the University of Massachusetts and the Infrared Processing and Analysis Center/California Institute of Technology, funded by the National Aeronautics and Space Administration and the National Science Foundation.

\section{Appendix A: X-ray emission in CMaR1}

\section{A.1. Sources detected in CMa R1}

The ROSAT PSPC $2^{\circ}$ image of Field 1 was obtained with an exposure of $20 \mathrm{ks}$ and pointed towards $\alpha=07^{\mathrm{h}} 01^{\mathrm{m}}, \delta=-11^{\circ} 24^{\prime}$ (J2000 coordinates). Due to a strong background emission of instrumental and cosmic origin in the spectral range $0.1-0.4 \mathrm{keV}$ (see Gregorio-Hetem et al. 1998), the sources were analyzed in the $0.4-2.4 \mathrm{keV}$ range. The X-ray data derived from the ROSAT image analysis is given in Table A.1, along with stellar identifications.

Sixty-one X-ray sources were detected, 48 of them having $S / N>3.5$, with $\mathrm{X}$-ray luminosities in the range of $6 \times$ $10^{30} \mathrm{erg} \mathrm{s}^{-1}$ to $8 \times 10^{32} \mathrm{erg} \mathrm{s}^{-1}$. Following Gregorio-Hetem et al. (1998) the approximate X-ray luminosities $\left(\tilde{L}_{\mathrm{X}}\right)$ were derived by using the correspondence between count-rate and $\mathrm{X}$-ray flux given by $1 \mathrm{cnt} \mathrm{ks}^{-1} \simeq 9 \times 10^{-15} \mathrm{erg} \mathrm{s}^{-1} \mathrm{~cm}^{-2}$. The assumed visual extinction, temperature and distance are respectively $A_{V}=1 \mathrm{mag}, k T_{\mathrm{X}}=1 \mathrm{keV}$, and $d \sim 1 \mathrm{kpc}$ for all the sources.

A similar procedure was adopted to obtain $\tilde{L}_{\mathrm{X}}$ for the sources detected in the ROSAT Field observed by Zinnecker \& Preibisch (1994). This other PSPC image was pointed to $\alpha=07^{\mathrm{h}} 03.7^{\mathrm{m}}$, $\delta=-11^{\circ} 33^{\prime}$ and had an exposure of $5 \mathrm{ks}$. We have identified 42 sources in this field, five of them coinciding with the sources identified in the first Field (described above). The range of X-ray luminosities in this sample is $10^{30} \mathrm{erg} \mathrm{s}^{-1}$ to $9.8 \times 10^{30} \mathrm{erg} \mathrm{s}^{-1}$.

\section{A.2. X-ray luminosity compared to optical and near-IR luminosities}

Figure A.1 shows the diagrams $L_{\mathrm{X}}$ versus $M_{R o}$ (dereddened absolute magnitude) of the sample, compared to the correlation of $\mathrm{X}$-ray emission with the optical absolute magnitude, expected 
Table A.1. X-ray sources detected in CMa R1 by ROSAT.

\begin{tabular}{|c|c|c|c|c|c|c|c|c|c|c|c|c|c|}
\hline CMaX & $\begin{array}{l}\text { Err } \\
\left({ }^{\prime \prime}\right)\end{array}$ & Cnt/ks & $S / N$ & $\log L_{\mathrm{x}}$ & $\begin{array}{c}\text { RA } \\
\mathrm{J} 2000 \\
\end{array}$ & $\begin{array}{c}\text { Dec } \\
\mathrm{J} 2000\end{array}$ & $\begin{array}{c}R \\
(\mathrm{mag}) \\
\end{array}$ & $\begin{array}{c}A_{V} \\
(\mathrm{mag})\end{array}$ & $\begin{array}{c}J \\
(\mathrm{mag}) \\
\end{array}$ & $\begin{array}{c}H \\
\text { (mag) }\end{array}$ & $\begin{array}{c}K \\
\text { (mag) } \\
\end{array}$ & $\begin{array}{c}\text { Age } \\
(\mathrm{Myr})\end{array}$ & $\begin{array}{l}\text { Mass } \\
\left(M_{\odot}\right) \\
\end{array}$ \\
\hline 1 & 30 & $16.7 \pm 1.6$ & 10.4 & 31.25 & 65752.6 & -112723 & 9.7 & 1.0 & 8.9 & 8.7 & 8.6 & $<1$ & $3.0-5.0$ \\
\hline 2 & 30 & $5.0 \pm 0.8$ & 6.3 & 30.72 & 65817.6 & -113710 & 15.7 & 1.0 & 14.5 & 13.9 & 13.8 & $05-10$ & $1.0-1.5$ \\
\hline $3^{a}$ & 30 & $17.9 \pm 1.6$ & 11.2 & 31.28 & 65842.6 & -114200 & 9.0 & 0.5 & 8.4 & 8.2 & 8.1 & $<1$ & $3.0-5.0$ \\
\hline $4^{b}$ & 30 & $19.4 \pm 1.4$ & 13.9 & 31.31 & 65902.0 & -110038 & 10.8 & 0.4 & 11.5 & 11.4 & 11.4 & 05-10 & $1.5-2.0$ \\
\hline $5^{c}$ & 30 & $5.0 \pm 0.9$ & 5.6 & 30.72 & 65910.7 & -114003 & 8.3 & 0.5 & 7.7 & 7.5 & 7.4 & $<1 ?$ & $>5$ \\
\hline 6 & 30 & $7.0 \pm 0.9$ & 7.8 & 30.87 & 65910.7 & -115730 & 13.0 & 1.0 & 11.2 & 10.8 & 10.7 & $02-05$ & $2.0-3.0$ \\
\hline 7 & 30 & $5.1 \pm 0.8$ & 6.4 & 30.73 & 65931.0 & -115818 & 11.8 & 0.9 & 11.0 & 10.6 & 10.6 & $02-05$ & $2.0-3.0$ \\
\hline 8 & 20 & $2.4 \pm 0.6$ & 4.0 & 30.40 & 65951.1 & -111552 & 12.7 & 0.5 & 11.2 & 10.7 & 10.5 & $01-02$ & $2.0-3.0$ \\
\hline 9 & 12 & $1.4 \pm 0.4$ & 3.5 & 30.17 & 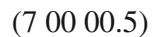 & $(-111409)$ & - & - & - & - & - & - & - \\
\hline 10 & 30 & $5.1 \pm 0.7$ & 7.3 & 30.73 & 70003.4 & -114745 & - & - & 9.6 & 9.6 & 9.6 & $<1$ & $3.0-5.0$ \\
\hline 11 & 10 & $2.8 \pm 0.5$ & 5.6 & 30.47 & 70003.8 & -111526 & 14.8 & 0.4 & 13.7 & 13.2 & 13.0 & $20-50$ & $1.0-1.5$ \\
\hline 12 & 5 & $3.7 \pm 0.6$ & 6.2 & 30.59 & 70005.2 & -113146 & 14.8 & 0.8 & 13.4 & 12.8 & 12.7 & $05-10$ & $1.0-1.5$ \\
\hline 13 & 12 & $1.2 \pm 0.3$ & 4.0 & 30.10 & 70008.0 & -113700 & 14.9 & 0.5 & 14.1 & 13.4 & 13.2 & $05-10$ & $1.0-1.5$ \\
\hline 14 & 12 & $2.2 \pm 0.4$ & 5.5 & 30.36 & 70012.5 & -113433 & 16.6 & 0.8 & 13.8 & 13.1 & 12.9 & $02-05$ & $1.0-1.5$ \\
\hline 15 & 10 & $1.9 \pm 0.4$ & 4.8 & 30.30 & $\left(\begin{array}{llll}7 & 00 & 14.5)\end{array}\right.$ & $\left(\begin{array}{llll}-11 & 18 & 51\end{array}\right)$ & - & - & - & - & - & - & - \\
\hline 16 & 10 & $2.0 \pm 0.5$ & 4.0 & 30.32 & 70017.0 & -110803 & 13.7 & 0.4 & 12.6 & 12.4 & 12.3 & $>50$ & $1.5-2.0$ \\
\hline 17 & 9 & $1.3 \pm 0.3$ & 4.3 & 30.14 & (7 $00 \begin{array}{lll}79.8)\end{array}$ & $\left(\begin{array}{lll}-11 & 23 & 20\end{array}\right)$ & - & - & - - & - & - & - & - \\
\hline 18 & 8 & $1.1 \pm 0.3$ & 3.7 & 30.06 & 70026.5 & -112633 & 14.9 & 0.8 & 13.1 & 12.4 & 12.2 & $02-05$ & $1.0-1.5$ \\
\hline 19 & 30 & $4.6 \pm 0.8$ & 5.8 & 30.69 & 70027.1 & -115126 & 13.4 & 0.8 & 12.4 & 12.1 & 12.0 & $10-20$ & $1.5-2.0$ \\
\hline $20^{d}$ & 5 & $62.7 \pm 1.9$ & 33.0 & 31.82 & 70037.6 & -111445 & 7.8 & 0.7 & 6.6 & 6.0 & 5.9 & $<1 ?$ & $>5$ \\
\hline 21 & 30 & $41.6 \pm 1.9$ & 21.9 & 31.64 & 70040.4 & -110435 & 12.3 & 0.9 & 10.6 & 9.9 & 9.7 & $<1$ & $1.0-1.5$ \\
\hline 22 & 6 & $2.0 \pm 0.4$ & 5.0 & 30.32 & (7 0041.3 ) & $(-113450)$ & - & - & - & - & - & - & - \\
\hline 23 & 5 & $3.4 \pm 0.5$ & 6.8 & 30.55 & 70043.4 & -111714 & 14.3 & 0.5 & 12.9 & 12.3 & 12.2 & $02-05$ & $1.5-2.0$ \\
\hline $24^{*}$ & 8 & $1.1 \pm 0.4$ & 2.8 & 30.06 & 70045.4 & -113100 & 16.9 & 0.5 & 15.9 & 15.6 & 15.0 & $? ?$ & $? ?$ \\
\hline 25 & 8 & $0.4 \pm 0.2$ & 2.0 & 29.62 & 70045.5 & -112029 & 17.0 & 0.8 & 16.0 & 15.7 & 15.6 & $? ?$ & $? ?$ \\
\hline 26 & 12 & $1.2 \pm 0.3$ & 4.0 & 30.10 & 70054.6 & -113412 & 14.0 & 0.8 & 13.2 & 12.8 & 12.7 & $20-50$ & $1.0-1.5$ \\
\hline $27^{*}$ & 10 & $2.2 \pm 0.5$ & 4.4 & 30.36 & 70055.5 & -112145 & 15.7 & 0.7 & 14.6 & 14.3 & 14.1 & $01-02$ & $0.1-0.5$ \\
\hline 28 & 8 & $0.9 \pm 0.3$ & 3.0 & 29.98 & 70055.8 & -112625 & 15.8 & 0.8 & 13.6 & 12.8 & 12.6 & $? ?$ & $? ?$ \\
\hline 29 & 5 & $2.5 \pm 0.4$ & 6.3 & 30.42 & 70100.8 & -111929 & 15.4 & 1.0 & 12.8 & 12.1 & 11.9 & $<1$ & $0.5-1.0$ \\
\hline 30 & 12 & $2.1 \pm 0.5$ & 4.2 & 30.34 & 70104.1 & -113948 & 16.4 & 0.7 & 15.2 & 14.4 & 14.2 & $05-10$ & $0.1-0.5$ \\
\hline 31 & 9 & $0.8 \pm 0.3$ & 2.7 & 29.93 & 70106.9 & -113123 & 10.6 & 0.7 & 9.4 & 9.0 & 8.8 & $<1$ & $3.0-5.0$ \\
\hline 32 & 8 & $2.1 \pm 0.4$ & 5.3 & 30.34 & 70111.2 & -112750 & 11.4 & 0.9 & 10.8 & 10.6 & 10.5 & $02-05$ & $2.0-3.0$ \\
\hline 33 & 15 & $0.9 \pm 0.3$ & 3.0 & 29.98 & 70113.1 & -112101 & 16.4 & 1.0 & 14.9 & 14.4 & 14.3 & $? ?$ & $? ?$ \\
\hline 34 & 8 & $1.3 \pm 0.3$ & 4.3 & 30.14 & 70116.3 & -112558 & 15.2 & 1.0 & 13.7 & 13.0 & 12.9 & $05-10$ & $1.0-1.5$ \\
\hline 35 & 8 & $0.7 \pm 0.3$ & 2.3 & 29.87 & 70120.1 & -111744 & 15.6 & 0.7 & 13.3 & 12.9 & 12.8 & $20-50$ & $1.0-1.5$ \\
\hline 36 & 15 & $1.1 \pm 0.3$ & 3.7 & 30.06 & 70124.4 & -110906 & 16.2 & 0.5 & 14.8 & 14.2 & 14.1 & $20-50$ & $0.5-1.0$ \\
\hline 37 & 15 & $2.1 \pm 0.4$ & 5.3 & 30.34 & $\left(\begin{array}{lll}7 & 01 & 25.0\end{array}\right)$ & $(-110850)$ & - & - & - & - & - & - & - \\
\hline 38 & 5 & $3.5 \pm 0.4$ & 8.8 & 30.57 & 70126.8 & -112821 & 13.5 & 0.8 & 12.3 & 12.0 & 11.9 & $10-20$ & $1.5-2.0$ \\
\hline 39 & 8 & $0.8 \pm 0.3$ & 2.7 & 29.93 & 70129.2 & -112130 & 15.4 & 0.8 & 14.1 & 13.5 & 13.5 & $10-20$ & $1.0-1.5$ \\
\hline 40 & 10 & $2.0 \pm 0.4$ & 5.0 & 30.32 & 70130.4 & -112229 & 16.9 & 1.2 & 15.4 & 14.8 & 14.8 & $? ?$ & $? ?$ \\
\hline 41 & 8 & $1.6 \pm 0.4$ & 4.0 & 30.23 & 70134.0 & -112533 & 15.3 & 0.8 & 13.8 & 13.2 & 13.1 & $10-20$ & $1.0-1.5$ \\
\hline 42 & 10 & $1.8 \pm 0.4$ & 4.5 & 30.28 & (7 $\left.\begin{array}{llll}7 & 01 & 34.3\end{array}\right)$ & $\left(\begin{array}{lll}-11 & 11 & 16\end{array}\right)$ & - & - & - & - & - & - & - \\
\hline 43 & 15 & $0.6 \pm 0.3$ & 2.0 & 29.80 & 070135.1 & -113638 & 14.3 & 1.3 & 12.1 & 11.8 & 11.7 & $10-20$ & $1.5-2.0$ \\
\hline 44 & 8 & $1.2 \pm 0.3$ & 4.0 & 30.10 & 70135.8 & -111736 & 13.6 & 0.8 & 12.8 & 12.5 & 12.4 & $10-20$ & $1.0-1.5$ \\
\hline 45 & 10 & $1.2 \pm 0.4$ & 3.0 & 30.10 & 70141.1 & -113257 & 12.9 & 1.0 & 12.2 & 11.9 & 11.9 & $10-20$ & $1.5-2.0$ \\
\hline 46 & 15 & $1.4 \pm 0.4$ & 3.5 & 30.17 & (7 0143.0$)$ & $\left(\begin{array}{lll}-11 & 10 & 20\end{array}\right)$ & - & - & - & - & - & - & - \\
\hline 47 & 8 & $2.1 \pm 0.4$ & 5.3 & 30.34 & 70145.9 & -113033 & 15.4 & 0.7 & 13.4 & 12.7 & 12.5 & $01-02$ & $0.5-1.0$ \\
\hline $48^{e}$ & 15 & $2.7 \pm 0.4$ & 6.8 & 30.45 & 70149.3 & -111807 & 7.5 & 1.7 & 6.3 & 6.2 & 6.0 & $? ?$ & $? ?$ \\
\hline 49 & 9 & $1.5 \pm 0.4$ & 3.8 & 30.20 & 70149.5 & -111641 & 12.2 & 1.7 & 10.8 & 10.3 & 10.2 & $02-05$ & $2.0-3.0$ \\
\hline 50 & 12 & $1.1 \pm 0.3$ & 3.7 & 30.06 & 70152.4 & -112018 & 13.4 & 1.3 & 12.1 & 11.7 & 11.5 & $05-10$ & $1.5-2.0$ \\
\hline 51 & 8 & $1.3 \pm 0.4$ & 3.3 & 30.14 & 70156.3 & -112407 & 16.5 & 1.2 & 14.3 & 13.6 & 13.4 & $10-20$ & $1.0-1.5$ \\
\hline 52 & 8 & $2.3 \pm 0.4$ & 5.8 & 30.38 & 70158.7 & -111545 & 13.5 & 1.7 & 12.3 & 11.6 & 11.5 & $01-02$ & $2.0-3.0$ \\
\hline 53 & 14 & $1.5 \pm 0.4$ & 3.8 & 30.20 & 70204.4 & -111944 & 14.1 & 1.2 & 13.4 & 13.0 & 12.9 & $>50$ & $05-1.0$ \\
\hline 54 & 15 & $0.5 \pm 0.3$ & 1.7 & 29.72 & 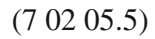 & $(-112650)$ & - & - & - & - & - & - & - \\
\hline 55 & 30 & $0.6 \pm 0.3$ & 2.0 & 29.80 & 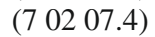 & $\left(\begin{array}{lll}-11 & 17 & 11\end{array}\right)$ & - & - & - & - & - & - & - \\
\hline 56 & 30 & $5.3 \pm 0.8$ & 6.6 & 30.75 & 70238.9 & -113039 & 12.8 & 0.9 & 9.9 & 8.9 & 8.6 & $? ?$ & $? ?$ \\
\hline $57^{f}$ & 30 & $2.3 \pm 1.0$ & 2.3 & 30.40 & 70242.6 & -112712 & 8.0 & 1.6 & 7.8 & 7.8 & 7.7 & $<1$ ? & $>5$ \\
\hline 58 & 30 & $2.0 \pm 1.0$ & 2.0 & 30.32 & 70247.3 & -112808 & 16.6 & 1.6 & 14.9 & 14.3 & 14.1 & $>50$ & $1.0-1.5$ \\
\hline 59 & 30 & $1.9 \pm 1.0$ & 2.0 & 30.31 & 70251.3 & -112403 & 17.5 & 4.0 & 14.6 & 13.7 & 13.4 & $05-10$ & $1.0-1.5$ \\
\hline
\end{tabular}

Columns description: (1) Source name: CMaX 1 to 56 (Field 1), CMaX 62 to 98 (Field 2), CMaX 57 to 61 (both fields); (2-5) X-ray data; $(6,7)$ Coordinates of the optical counterpart, when available; $(8,9) R$ magnitude and visual extinction $\left(A_{V}\right)$, obtained from available catalogues; (10-12) Near-IR magnitudes from the 2MASS catalogue; $(13,14)$ The estimation of age and mass is based on near-IR.

Notes: Stars previously identified (Clariá 1974, indicated by "C and Shevchenko et al. 1999, indicated by "SEI"): (a) HD 51896 (F5), (b) SS 75, (c) HD 52014 (F5), (d) HD 52385 (K0), (e) GU CMa, HD 52721 (B2V), C 63, SEI 158; (f) FZ CMa (B2.5), C 67, SEI 159; (g) Z CMa (Bpe), C 76, SEI 161; (h) BD -111762 (B2), C 77, SEI 92; (i) C 14, SEI 94. Members of clusters: (j) vdB-RN92, (k) BRC 27. Objects detected by $X M M-N e w t o n$ and/or Chandra are indicated by "x". Relevant sources: $(*) H-K$ excess. 
Table A.1. continued.

\begin{tabular}{|c|c|c|c|c|c|c|c|c|c|c|c|c|c|}
\hline CMaX & $\begin{array}{l}\text { Err } \\
\left({ }^{\prime \prime}\right)\end{array}$ & Cnt/ks & $\overline{S / N}$ & $\log L_{\mathrm{x}}$ & $\begin{array}{c}\text { RA } \\
\text { J2000 }\end{array}$ & $\begin{array}{c}\text { Dec } \\
\text { J2000 }\end{array}$ & $\begin{array}{c}R \\
(\mathrm{mag})\end{array}$ & $\begin{array}{c}A_{V} \\
\text { (mag) }\end{array}$ & $\begin{array}{c}J \\
(\mathrm{mag})\end{array}$ & $\begin{array}{c}H \\
(\mathrm{mag})\end{array}$ & $\begin{array}{c}K \\
(\mathrm{mag})\end{array}$ & $\begin{array}{c}\text { Age } \\
\text { (Myr) }\end{array}$ & $\begin{array}{l}\text { Mass } \\
\left(M_{\odot}\right)\end{array}$ \\
\hline 60 & 30 & $1.5 \pm 0.9$ & 1.6 & 30.20 & 70330.4 & -114807 & 13.7 & 2.4 & 13.5 & 13.0 & 12.8 & $20-50$ & $1.0-1.5$ \\
\hline $61^{x}$ & 30 & $1.7 \pm 0.9$ & 1.9 & 30.27 & 70333.5 & -113427 & 15.2 & 2.6 & 13.5 & 12.8 & 12.5 & $05-10$ & $1.0-1.5$ \\
\hline $62^{x}$ & 30 & $1.5 \pm 0.9$ & 1.7 & 30.20 & 70342.1 & -113514 & 15.5 & 2.5 & 13.4 & 12.6 & 12.4 & $02-05$ & $1.5-2.0$ \\
\hline $63^{*, g, x}$ & 30 & $2.5 \pm 1.0$ & 2.5 & 30.43 & 70343.2 & -113306 & 9.1 & 3.4 & 6.5 & 5.2 & 3.8 & $? ?$ & ?? \\
\hline $64^{x}$ & 30 & $2.9 \pm 1.1$ & 2.7 & 30.49 & 70347.5 & -113149 & 13.8 & 1.3 & 13.5 & 12.8 & 12.6 & $01-02$ & $0.5-1.0$ \\
\hline 65 & 30 & $1.2 \pm 0.8$ & 1.5 & 30.11 & 70348.2 & -114146 & 14.0 & 0.3 & 13.6 & 12.8 & 12.6 & $? ?$ & $? ?$ \\
\hline $66^{j, x}$ & 30 & $6.2 \pm 1.4$ & 4.3 & 30.82 & 70351.5 & -113456 & - & - & 11.0 & 10.8 & 10.7 & $01-02$ & $3.0-5.0$ \\
\hline $67^{x}$ & 30 & $2.1 \pm 1.0$ & 2.2 & 30.35 & 70352.5 & -112617 & 16.0 & 1.4 & 13.7 & 12.9 & 12.6 & $? ?$ & $? ?$ \\
\hline $68^{j}$ & 30 & $2.0 \pm 1.0$ & 2.1 & 30.34 & 70354.0 & -113237 & 14.1 & 2.3 & 14.6 & 13.7 & 13.3 & $2-5$ & $0.5-1.0$ \\
\hline $69^{h}$ & 30 & $7.9 \pm 1.6$ & 4.9 & 30.93 & 70354.4 & -112829 & 9.4 & 1.5 & 9.4 & 9.4 & 9.4 & $<1$ ? & $>5$ \\
\hline 70 & 30 & $2.2 \pm 1.0$ & 2.2 & 30.37 & 70354.8 & -114237 & 15.1 & 0.3 & 12.0 & 11.5 & 11.3 & $01-02$ & $1.5-2.0$ \\
\hline $71^{x}$ & 30 & $4.9 \pm 1.3$ & 3.7 & 30.72 & 70355.7 & -112932 & 14.9 & 2.5 & 12.9 & 12.2 & 12.0 & $02-05$ & $1.5-2.0$ \\
\hline $72^{i, j}$ & 30 & $1.6 \pm 0.9$ & 1.9 & 30.25 & 70356.8 & -113442 & 11.0 & 7.0 & 10.5 & 10.2 & 10.1 & $? ?$ & ?? \\
\hline $73^{j, x}$ & 30 & $2.9 \pm 1.1$ & 2.7 & 30.50 & 70400.4 & -113400 & 12.7 & 7.0 & 12.1 & 10.9 & 10.2 & $<1$ & $2.0-3.0$ \\
\hline $74^{*, k}$ & 30 & $9.2 \pm 1.8$ & 5.3 & 31.00 & 70401.4 & -112335 & 12.6 & 2.5 & 11.5 & 10.8 & 10.3 & $02-05$ & $2.0-3.0$ \\
\hline $75^{k, l, x}$ & 30 & $6.4 \pm 1.5$ & 4.3 & 30.84 & 70402.3 & -112539 & 10.8 & 1.3 & 10.4 & 10.3 & 10.3 & $1-2$ & $3.0-5.0$ \\
\hline $76^{m}$ & 30 & $3.8 \pm 1.3$ & 3.0 & 30.61 & 70406.6 & -111835 & 10.9 & 6.0 & 13.1 & 12.2 & 11.6 & $02-05$ & $2.0-3.0$ \\
\hline $77^{m}$ & 30 & $3.0 \pm 1.1$ & 2.7 & 30.51 & 70409.2 & -113008 & 14.2 & 0.8 & 13.7 & 13.0 & 12.9 & $01-02$ & $1.0-1.5$ \\
\hline 78 & 30 & $2.2 \pm 1.2$ & 1.9 & 30.38 & 70411.3 & -111648 & 13.4 & 3.5 & 12.0 & 11.4 & 11.1 & $05-10$ & $2.0-3.0$ \\
\hline 79 & 30 & $4.1 \pm 1.3$ & 3.2 & 30.64 & 70412.1 & -112110 & 13.8 & 2.9 & 14.4 & 13.4 & 13.1 & $? ?$ & $? ?$ \\
\hline 80 & 30 & $2.1 \pm 1.0$ & 2.1 & 30.36 & 70412.3 & -113923 & 12.0 & 1.2 & 11.3 & 11.0 & 11.0 & $05-10$ & $2.0-3.0$ \\
\hline $81^{m, n}$ & 30 & $3.2 \pm 1.2$ & 2.7 & 30.54 & 70413.2 & -111901 & 7.8 & 3.5 & 10.9 & 10.6 & 10.5 & $01-02$ & $3.0-5.0$ \\
\hline $82^{o, x}$ & 30 & $3.8 \pm 1.2$ & 3.0 & 30.61 & 70415.9 & -112406 & 9.4 & 1.4 & 9.0 & 8.9 & 8.9 & $<1 ?$ & $>5$ \\
\hline $83^{p}$ & 30 & $3.5 \pm 0.8$ & 4.3 & 30.58 & 70417.4 & -114310 & 10.9 & 0.6 & 10.2 & 10.1 & 10.0 & $02-05$ & $3.0-5.0$ \\
\hline $84^{x}$ & 30 & $3.5 \pm 0.8$ & 4.3 & 30.58 & 70418.3 & -114236 & 13.8 & 0.0 & 12.0 & 11.5 & 11.3 & $02-05$ & $1.5-2.0$ \\
\hline 85 & 30 & $4.2 \pm 1.3$ & 3.3 & 30.66 & 70418.8 & -112447 & 14.7 & 0.8 & 14.6 & 13.9 & 13.7 & $05-10$ & $0.5-1.0$ \\
\hline $86^{x}$ & 30 & $2.9 \pm 1.1$ & 2.6 & 30.50 & 70419.1 & -113348 & 13.4 & 0.4 & 13.1 & 12.4 & 12.2 & $02-05$ & $0.5-1.0$ \\
\hline 87 & 30 & $3.0 \pm 1.2$ & 2.6 & 30.51 & 70420.0 & -112222 & 13.2 & 0.9 & 14.4 & 13.3 & 12.7 & $? ?$ & $? ?$ \\
\hline 88 & 30 & $1.8 \pm 0.9$ & 1.9 & 30.29 & 70420.6 & -113644 & 16.7 & 0.7 & 14.6 & 13.7 & 13.5 & $? ?$ & $? ?$ \\
\hline $89^{x}$ & 30 & $3.5 \pm 1.2$ & 2.9 & 30.57 & 70426.3 & -113121 & 13.8 & 0.8 & 12.5 & 11.6 & 11.2 & ?? & ?? \\
\hline 90 & 30 & $4.0 \pm 1.3$ & 3.1 & 30.63 & 70425.3 & -113428 & 13.2 & 1.1 & 11.7 & 11.1 & 10.9 & $<1$ & $1.5-2.0$ \\
\hline $91^{x}$ & 30 & $5.2 \pm 1.7$ & 3.1 & 30.75 & 70429.8 & -114721 & 12.1 & 0.0 & 11.2 & 10.5 & 10.3 & $? ?$ & $? ?$ \\
\hline $92^{x}$ & 30 & $2.3 \pm 1.1$ & 2.2 & 30.39 & 70431.0 & -113242 & 14.0 & 0.8 & 13.9 & 13.1 & 12.9 & $01-02$ & $0.1-0.5$ \\
\hline 93 & 30 & $2.8 \pm 1.1$ & 2.4 & 30.47 & 70437.0 & -113059 & 14.0 & 0.0 & 14.0 & 13.8 & 13.7 & $? ?$ & $? ?$ \\
\hline 94 & 30 & $1.6 \pm 1.0$ & 1.5 & 30.23 & 70448.4 & -111507 & 15.7 & 0.4 & 14.8 & 14.4 & 14.1 & $>50$ & $1.0-1.5$ \\
\hline 95 & 30 & $5.4 \pm 1.8$ & 3.0 & 30.76 & 70452.5 & -113821 & 14.7 & 0.2 & 13.2 & 12.4 & 12.1 & ?? & ?? \\
\hline $96^{x}$ & 30 & $3.4 \pm 1.5$ & 2.3 & 30.56 & 70456.3 & -112933 & 12.5 & 1.2 & 11.5 & 10.9 & 10.2 & 01-02 & $2.0-3.0$ \\
\hline 97 & 30 & $0.9 \pm 0.6$ & 1.7 & 30.00 & 70515.5 & -113106 & 14.8 & 0.0 & 13.2 & 12.8 & 12.7 & $20-50$ & $1.0-1.5$ \\
\hline 98 & 30 & $0.9 \pm 0.6$ & 1.7 & 30.00 & 70515.7 & -113205 & 14.4 & 0.0 & 12.8 & 12.1 & 12.0 & $01-02$ & $1.0-1.5$ \\
\hline
\end{tabular}

Notes: Stars previously identified (Clariá 1974, indicated by "C" and Shevchenko et al. 1999, indicated by "SEI"): (1) SEI 99; (n) SEI 111; (o) HD 53339 (B3), C84, SEI114; (p) HD 53396 (B9), SEI 112. Members of clusters: (k) BRC 27; (m) NGC 2327. Objects detected by $X M M-N e w t o n$ and/or Chandra are indicated by " $\mathrm{x}$ " (see list in Table A.2).

Table A.2. List of ROSAT sources observed by XMM-Newton or Chandra.

\begin{tabular}{ccccc}
\hline \hline CMaX & 2MASS & Source $^{a}$ & RA $^{b}$ & Dec $^{b}$ \\
\hline 61 & $07033347-1134269$ & C20 & 070333.46 & -113426.7 \\
62 & $07034209-1135139$ & C38 & 070342.07 & -113513.7 \\
63 & $07034316-1133062$ & C40 & 070343.13 & -113306.5 \\
64 & $07034751-1131489$ & C47 & 070347.50 & -113148.6 \\
66 & $07035152-1134557$ & C54 & 070351.50 & -113455.7 \\
67 & $07035249-1126168$ & C57 & 070352.38 & -112617.7 \\
71 & $07035575-1129315$ & C66 & 070355.73 & -112931.3 \\
73 & $07040041-1133596$ & C77 & 070400.35 & -113359.2 \\
75 & $07040234-1125393$ & X41 & 070402.48 & -112537.5 \\
82 & $07041588-1124055$ & X54 & 070415.94 & -112404.0 \\
84 & $07041833-1142359$ & X09 & 070418.42 & -114233.9 \\
86 & $07041912-1133480$ & X14 & 070419.21 & -113348.8 \\
89 & $07042625-1131207$ & X11 & 070426.35 & -113120.0 \\
91 & $07042982-1147208$ & X06 & 070429.97 & -114719.9 \\
92 & $07043099-1132417$ & X32 & 070431.11 & -113238.9 \\
96 & $07045632-1129332$ & X13 & 070456.44 & -112932.0 \\
\hline
\end{tabular}

Note: (a) Source number in the Chandra (C) or XMM-Newton (X) images; (b) respective J2000 coordinates. Positional deviations from the coordinates of near-IR counterparts (2MASS) are less than 2 arcsec for Chandra sources and less than 3.3 arcsec for XMM-Newton sources. for young stars. This correlation was obtained by comparing the magnitudes to the mean X-ray luminosities $\left(\tilde{L}_{\mathrm{X}}\right)$ estimated for ROSAT sources detected in the Chamaeleon I (Cha I) cloud, adopting the conversion $1 \mathrm{cnt} \mathrm{ks}^{-1}=3 \times 10^{28} \mathrm{erg} \mathrm{s}^{-1}$ (Feigelson et al. 1993).

Taking into account that the extinction cross-section at $2 \mu \mathrm{m}$ and at $2 \mathrm{keV}$ are almost the same (e.g., Ryter 1996), the X-ray emission was compared to the $J$ band magnitude in order to check whether these data show the same correlation as previously found for young, low-mass stars, i.e., the $\log \left(\tilde{L}_{\mathrm{X}}\right)$ versus $M_{J o}$ diagram (Casanova et al. 1995), which is presented in Fig. A.2. The Cha I sources were also plotted for comparison: since they are much closer $(d=140 \mathrm{pc})$ than CMa R1, the same ROSAT sensitivity allows to probe fainter, lower-mass young stars.

The absolute magnitudes of the Cha I sources were estimated by adopting a distance modulus of 5.73 and were dereddened by using their visual extinctions, when available. Otherwise, $A_{V}=1 \mathrm{mag}$ was adopted. The Cha I correlation was established on the basis of 16 sources with well-determined magnitudes and extinctions (see Casanova et al. 1995, for 


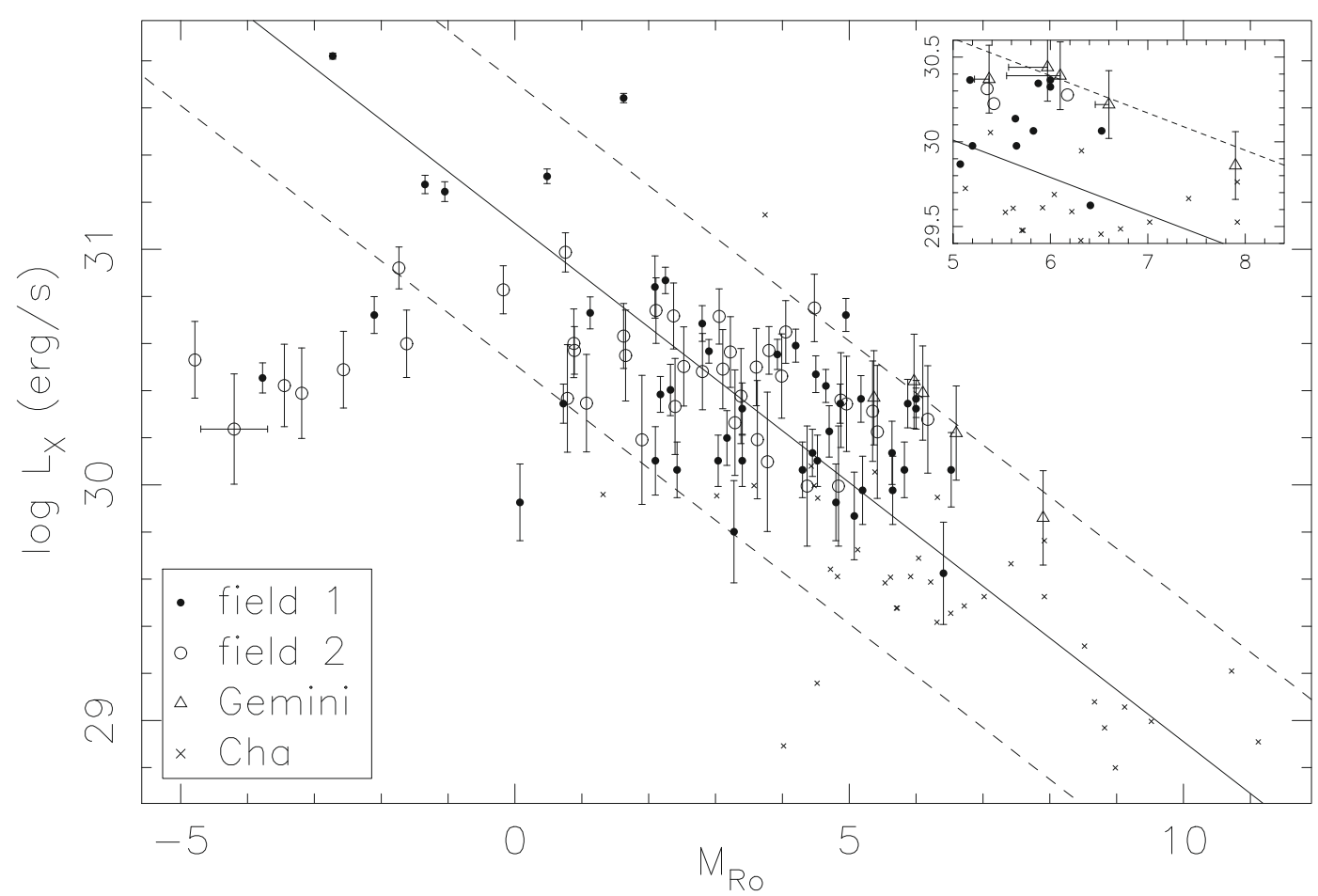

Fig. A.1. The distribution of the CMaX sources (circles) in the diagram of $\log \left(L_{\mathrm{X}}\right)$ as a function of de-reddened absolute magnitudes $M_{R o}$. Error bars of the optical magnitudes are shown for one illustrative example. Triangles indicate the five counterparts detected by Gemini, for which details are shown in the corner box. In this case, the horizontal bars show the possible range of magnitudes, when one or all counterparts contribute to the integrated X-ray luminosity (see text, Sect. 4.4). The Cha I X-ray data, represented by crosses, were extracted from Feigelson et al. (1993). The full line indicates the correlation obtained for the Cha I sources, which have well determined $L_{\mathrm{X}}$ and extinction. Dashed lines are used to indicate the $2 \sigma$ deviation from the linear fit.

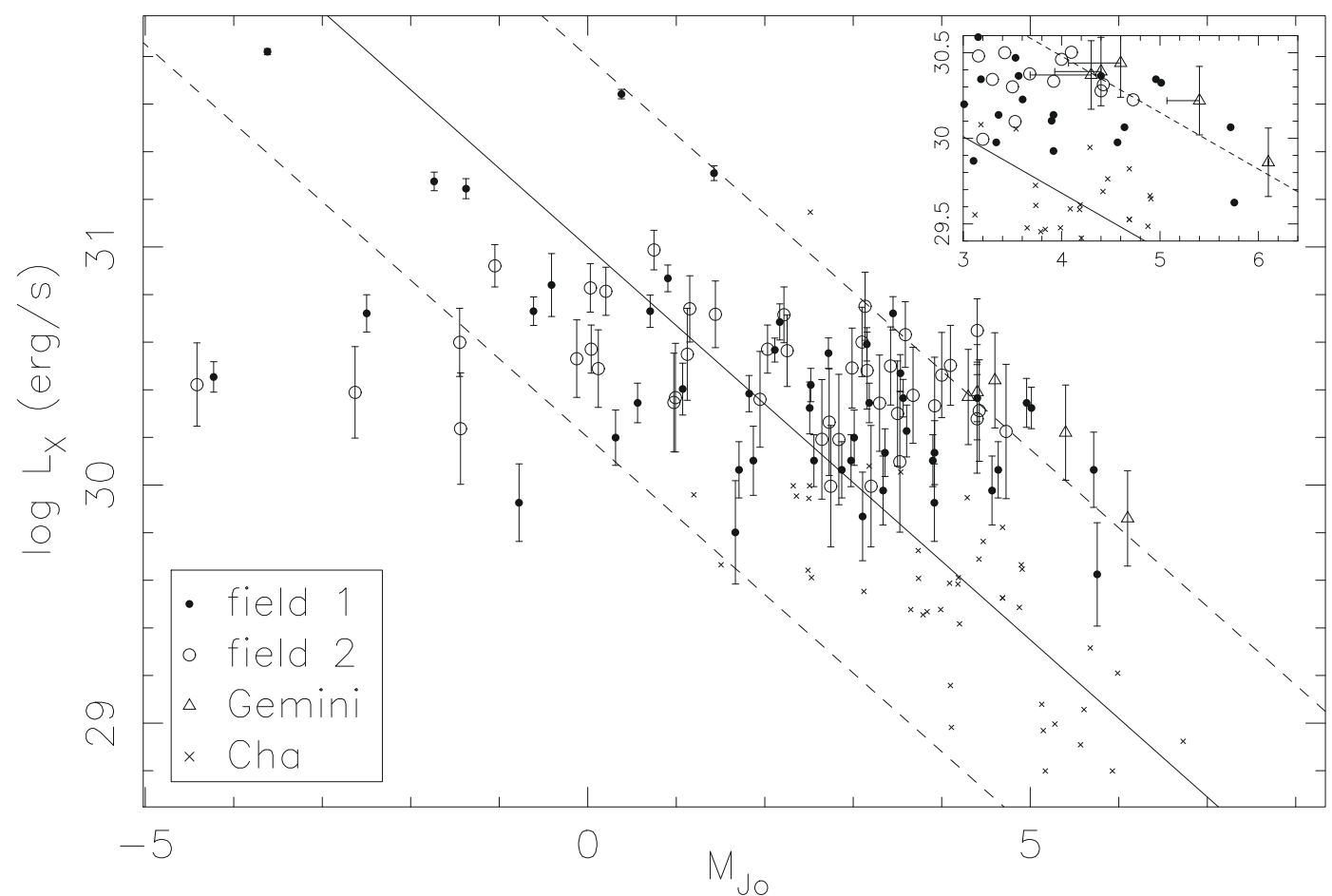

Fig. A.2. The same as Fig. A.1, showing the diagram of $\log \left(L_{\mathrm{X}}\right)$ as a function of de-reddened absolute magnitudes $M_{J o}$. Error bars for $J$ magnitudes are not plotted, since they are smaller than the symbols for most of the sources.

details; also Lawson et al. 1996). Figures A.1 and A.2 show the distribution of the Cha I sources and the corresponding linear regression: $\log \left(\tilde{L}_{X}\left[\mathrm{erg} \mathrm{s}^{-1}\right]\right)=31.10( \pm 0.3)-0.22 M_{R o}$, and for the $J$ band $\log \left(\tilde{L}_{X}\left[\mathrm{erg} \mathrm{s}^{-1}\right]\right)=31.00( \pm 0.4)-0.33 M_{J o}$. Dashed lines are used to indicate the $2 \sigma$ deviation.
Both diagrams, $\log \left(\tilde{L}_{\mathrm{X}}\right)$ vs. $M_{J o}$, and $\log \left(\tilde{L}_{\mathrm{X}}\right)$ vs. $M_{R o}$, show that total absolute magnitudes are compatible with the X-ray luminosities typical of young stars, but tend to deviate from the Cha I correlation as the counterparts become brighter. Also, in the upper right-hand box of the diagrams, we zoom on the Gemini counterparts. For these sources, the Gemini points 

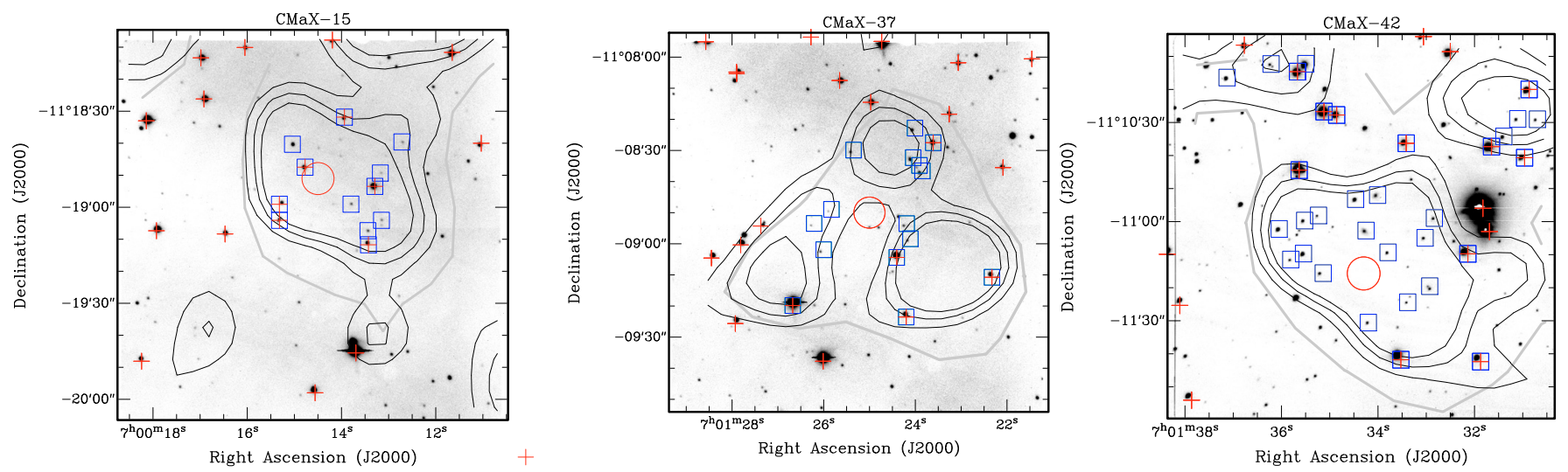

Fig. B.1. Optical image (Gemini I band) of CMaX-15 (left), 37 (middle), and 42 (right). The same as Fig. 6.
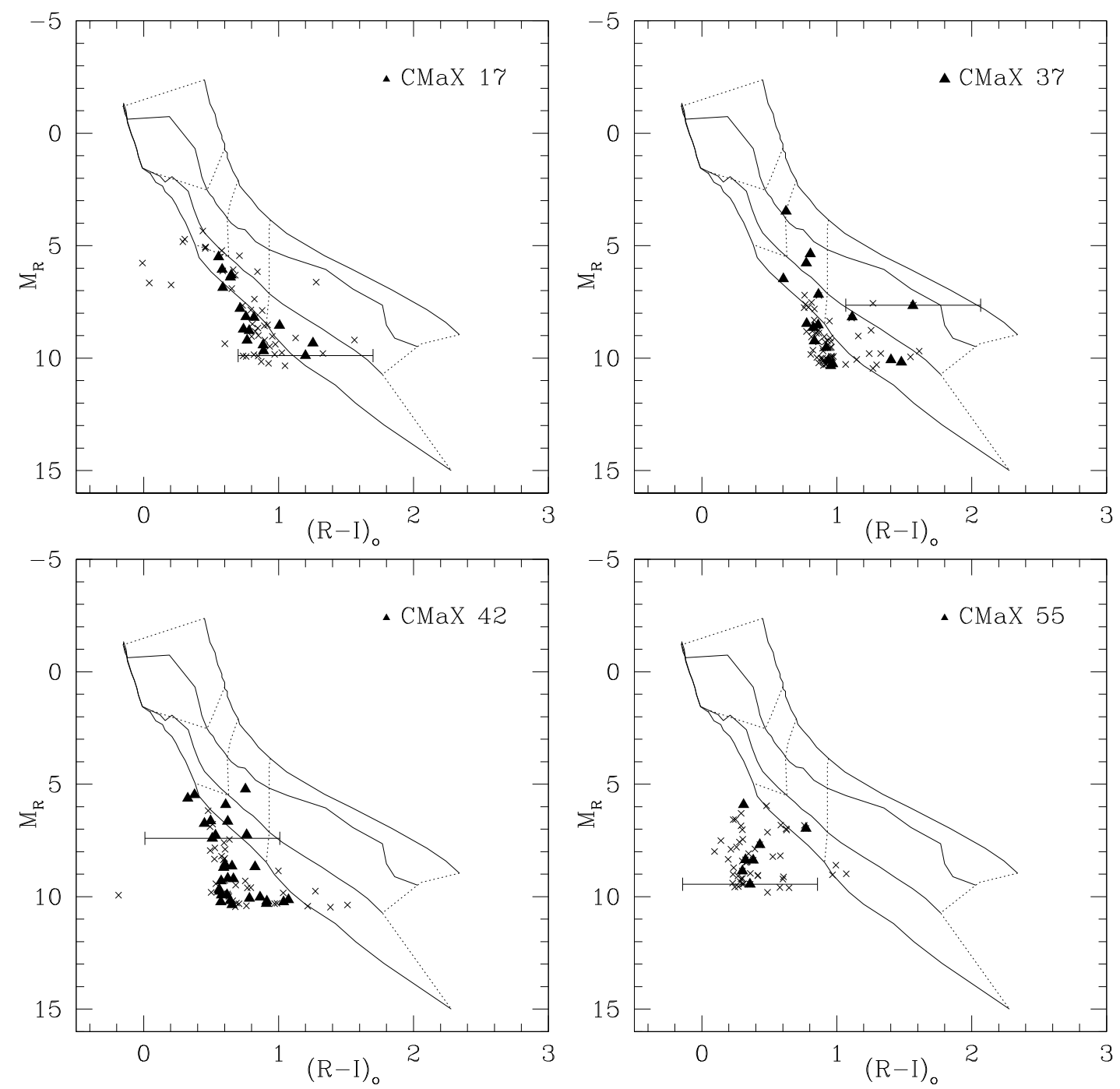

Fig. B.2. Colour-magnitude diagram of the optical candidates to be counterparts of the X-ray sources CMaX-17, 37, 42, 55. The same as Fig. 8.

are above the correlation, indicating that other, undetected (absorbed?) stars may also contribute to the unresolved X-ray emission. We defer a more detailed analysis of these diagrams to a later study.

\section{Appendix B: Results of the Gemini observations}

In this Appendix we show the continuation of the figures presented in the body of the paper.

\section{References}

Albacete Colombo, J. F., Méndez, M., \& Morrell, N. I. 2003, MNRAS, 346, 704 Bessell, M. S., Castelli, F., \& Plez, B. 1998, A\&A, 333, 231

Cambrésy, L., Beichman, C. A., Jarrett, T. H., \& Cutri, R. M. 2002, AJ, 123, 2559

Cardelli, J. A., Clayton, G. C., \& Mathis, J. S. 1989, ApJ, 345, 245

Casanova, S., Montmerle, T., Feigelson, E. D., \& André, P. 1995, ApJ, 439, 752 Dahm, S. E., \& Hillenbrand, L. A. 2007, AJ, 133, 2072

Deharveng, L., Lefloch, B., Kurtz, S., et al. 2008, A\&A, 482, 585

Dobashi, K., Uehara, H., Kandori, R., et al., 2005, PASJ, 57, SP1 
Elmegreen, B. G., \& Lada, C. J. 1977, ApJ, 214, 725

Favata, F., \& Micela, G. 2003, SSRv, 108, 577

Feigelson, E. D., \& Montmerle, T. 1999, ARA\&A, 37, 363

Feigelson, E. D., Casanova, S., Montmerle, T., \& Guibert, J. 1993, ApJ, 416, 623

Feigelson, E. D., Broos, P., Gaffney, J. A. III, et al. 2002, ApJ, 574, 258

Feigelson, E. D., Gaffney, J. A. III, Garmire, G., et al. 2003, ApJ, 584, 911

Flaccomio, E., Damiani, F., Micela, G., et al. 2003, ApJ, 582, 398

Garmire, G., Feigelson, E. D., Brooks, P., et al. 2000, AJ, 120, 1426

Getman, K. V., Flaccomio, E., Broos, P. S., et al. 2005a, ApJS, 160, 319

Getman, K. V., Feigelson, E. D., Grosso, N., et al. 2005b, ApJS, 160, 353

Gregorio-Hetem, J., Montmerle, T., Feigelson, E. D., \& Casanova, S. 1998, A\&A, 331, 193

Gregorio-Hetem, J., Montmerle, T., \& Marciotto, E. 2003, in Open Issues in Local Star Formation, ed. J. Lépine, \& J. Gregorio-Hetem (Kluwer), 193

Güdel, M. 2004, A\&AR, 12, 71

Güdel, M., Briggs, K. R., Arzner, K., et al. 2007, A\&A, 468, 353

Güdel, M., Briggs, K. R., Montmerle, T., et al. 2008, Science, 319, 309

Herbst, W., \& Assousa, G. E. 1977, ApJ, 217, 473

Jeffries, R. D., Naylor, T., Walter, F. M., Pozzo, M. P., \& Devey, C. R. 2009, MNRAS, 393, 538

Kaltcheva, N. T., \& Hilditch, R. W. 2000, MNRAS, 312, 753

Kidger, M. R. 2003, A\&A, 408, 767

Kohno, M., Koyama, K., \& Hamaguchi, K. 2002, ApJ, 567, 423 (Erratum in ApJ, 580, 626)

Kim, B. G., Kawamura, A., Yonekura, Y., \& Fukui, Y. 2004, PASJ, 56, 313

Lawson, W. A., Feigelson, E. D., \& Huenemoerder, D. P. 1996, MNRAS, 280, L1071

Linsky, J. L., Gagné, M., Mytyk, A., McCaughrean, M., \& Andersen, M. 2007, ApJ, 654, 347
López-Santiago, J., \& Caballero, J. A. 2008, A\&A, 491, 961

May, J., Murphy, D. C., \& Thaddeus, P. 1988, A\&AS, 73, 51

Monet, D. G., Levine, S. E., Canzian, B., et al. 2003, AJ, 125, 984

Ozawa, H., Grosso, N., \& Montmerle, T. 2005, A\&A, 429, 963

Preibisch, T., \& Zinnecker, H. 1999, AJ, 117, 2381

Pyatunina, T. B., \& Taraskin, Yu. M. 1986, Astron. Zhurnal, 63, 1098

Rauw, G., Nazé, Y., Gosset, E., et al. 2002, A\&A, 395, 499

Rojas, G., Gregorio-Hetem, J., Grosso, N., \& Montmerle, T. 2006, in Proceedings The X-ray Universe 2005, ESA Publications Division, 107

Ruprecht, J. 1966, IAU Trans., 12B, 348

Ryter, C. 1996, Ap\&SS, 236, 285

Sharpless, S. 1959, ApJS, 4, 257

Shevchenko, V. S., Ezhkova, O. V., Ibrahimov, M. A., et al. 1999, MNRAS, 310, 210

Snowden, S. L., Egger, R., Freyberg, M. J., et al. 1997, ApJ, 485, 125

Soares, J. B., \& Bica, E. 2002, A\&A, 388, 172

Soares, J. B., \& Bica, E. 2003, A\&A, 404, 217

Skrutskie, M. F., Cutri, R. M., Stiening, R., et al. 2006, The Two-Micron All Sky Survey (2MASS), AJ, 131, 1163

Stelzer, B., Flaccomio, E., Montmerle, T., et al. 2005, ApJS, 160, 557

Terranegra, L., Chavarria, K. C., Diaz, S., \& Gonzales-Patino, D. 1994, A\&AS, 104, 557

Townsley, L. K., Feigelson, E. D., Montmerle, T., et al. 2003, ApJ, 593, 874

Vuong, M. H., Montmerle, T., Grosso, N., et al. 2003, A\&A, 408, 581

Wang, J., Townsley, L. K., Feigelson, E. D., et al. 2008, ApJ, 675, 464

Wang, J., Feigelson, E. D., Townsley, L. K., et al. 2009, ApJ, 696, 47

Wolk, S. J., Bourke, T. L., Smith, R. K., Spitzbart, B., \& Alves, J. 2002, ApJ, 580, L161

Zavagno, A., Pomarès, M., Deharveng, L., et al. 2007, A\&A, 472, 835

Zinnecker, H., \& Preibisch, T. 1994, A\&A, 292, 152 